\title{
Is Isoeugenol a Prehapten? Characterization of a Thiol- Reactive Oxidative Byproduct of Isoeugenol and Potential Implications for Skin Sensitization.
}

\author{
Jongmin Ahn ${ }^{\dagger}$, Cristina Avonto ${ }^{\dagger *}$, Amar G. Chittiboyina ${ }^{\dagger}$ and Ikhlas A. Khan ${ }^{\dagger \dagger}$ \\ ${ }^{\dagger}$ National Center for Natural Products Research, Research Institute of Pharmaceutical \\ Science, School of Pharmacy, The University of Mississippi, University, Mississippi 38677, \\ United States \\ ¥Division of Pharmacognosy, Department of BioMolecular Sciences, School of Pharmacy, \\ The University of Mississippi, University, Mississippi 38677, United States.
}

\section{Supporting Information}

* Phone: +1 662915 1090, Fax: +1 662915 7989. Email: cavonto@olemiss.edu 


\section{Table of Contents}

Figure S1. TLC of aged isoeugenol mixture after reaction with DCYA. .............................................. 3

Figure S2. LC-MS of aged isoeugenol mixture after reaction with DCYA in pH10 buffer.................... S3

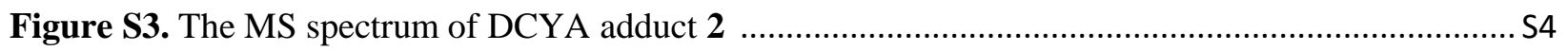

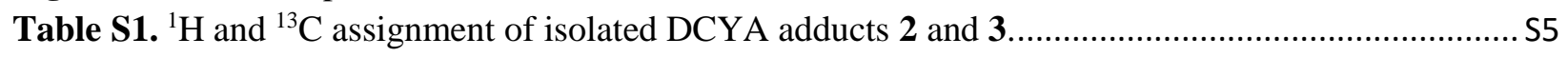

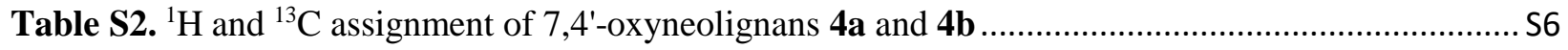

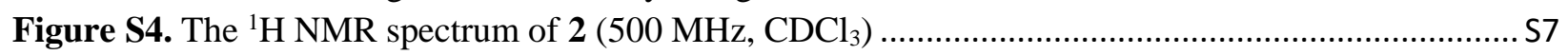

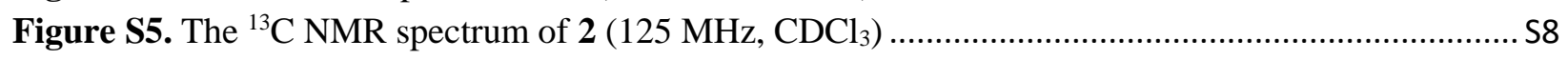

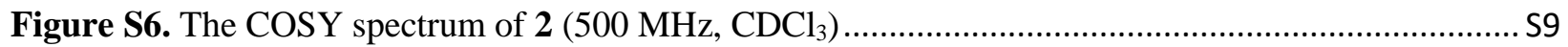

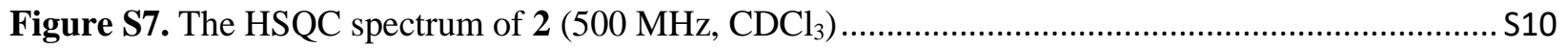

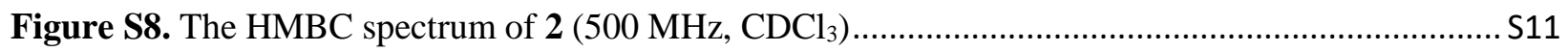

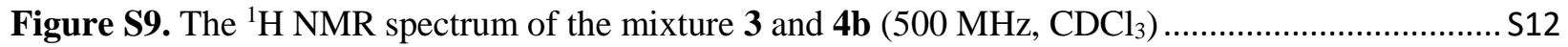

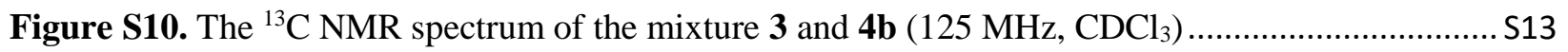

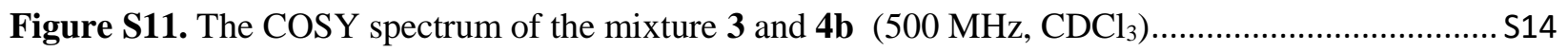

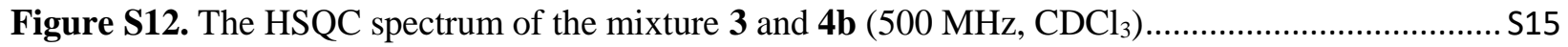

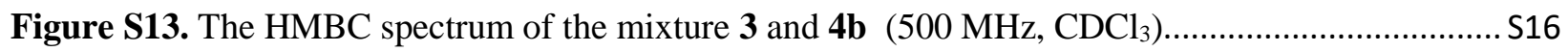

Figure S14. Failed attempts to synthesize isoeugenol epoxide ...................................................... S17

Figure S15. The ${ }^{1} \mathrm{H}$ NMR spectrum of aged isoeugenol mixture $\left(500 \mathrm{MHz}, \mathrm{CDCl}_{3}\right)$........................ S18

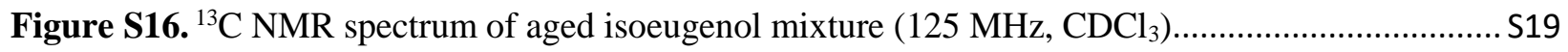

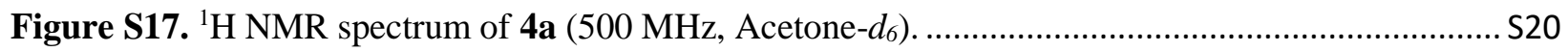

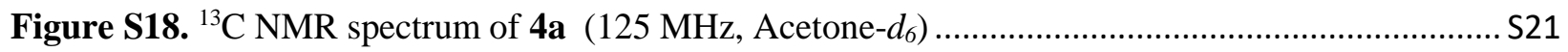

Figure S19. The COSY NMR spectrum of $4 \mathbf{a}\left(500 \mathrm{MHz}\right.$, Acetone- $\left.d_{6}\right)$............................................. S22

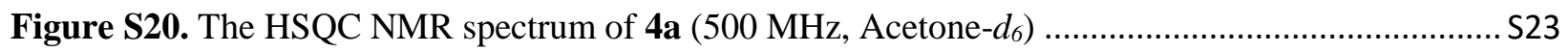

Figure S21. The HMBC NMR spectrum of $4 \mathbf{a}\left(500 \mathrm{MHz}\right.$, Acetone- $\left.d_{6}\right)$........................................... S24

Figure S22. Arrayed ${ }^{1} \mathrm{H}$ NMR spectra for 4a, DCYA, before addition of DBN (500 MHz, $\left.\mathrm{CDCl}_{3}\right)$...... S25

Figure S23. Arrayed ${ }^{1} \mathrm{H}$ NMR spectra for $4 \mathbf{a}$ and DCYA, after addition of DBN (500 MHz, $\mathrm{CDCl}_{3}$ )... S26

Table S3. Select normalized integral values before addition of DBN............................................... S27

Table S4. Select normalized integral values after addition of DBN................................................... S28 
Figure S1. TLC of the aged isoeugenol mixture after reaction with DCYA. TLC silica gel 60 without fluorescence indicator, hexane : acetone $6: 4 \mathrm{v} / \mathrm{v}, \mathrm{UV} 254 \mathrm{~nm}$

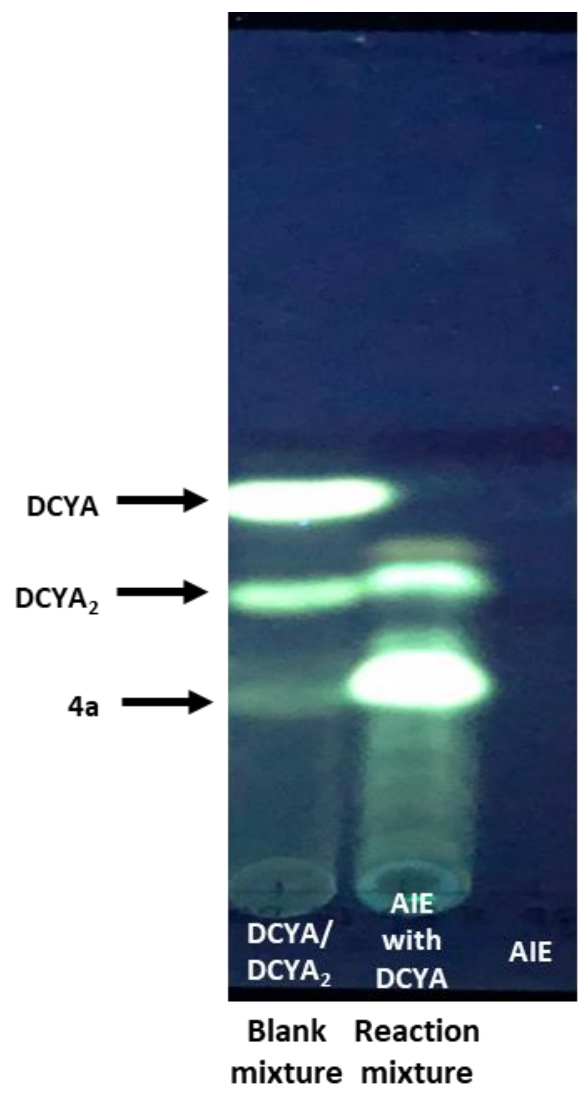

Figure S2. LC-MS of the aged isoeugenol mixture after reaction with DCYA in pH10 buffer. A major adduct 2 was $\mathrm{m} / \mathrm{z} 491$ at $11.23 \mathrm{~min}$ (positive mode).

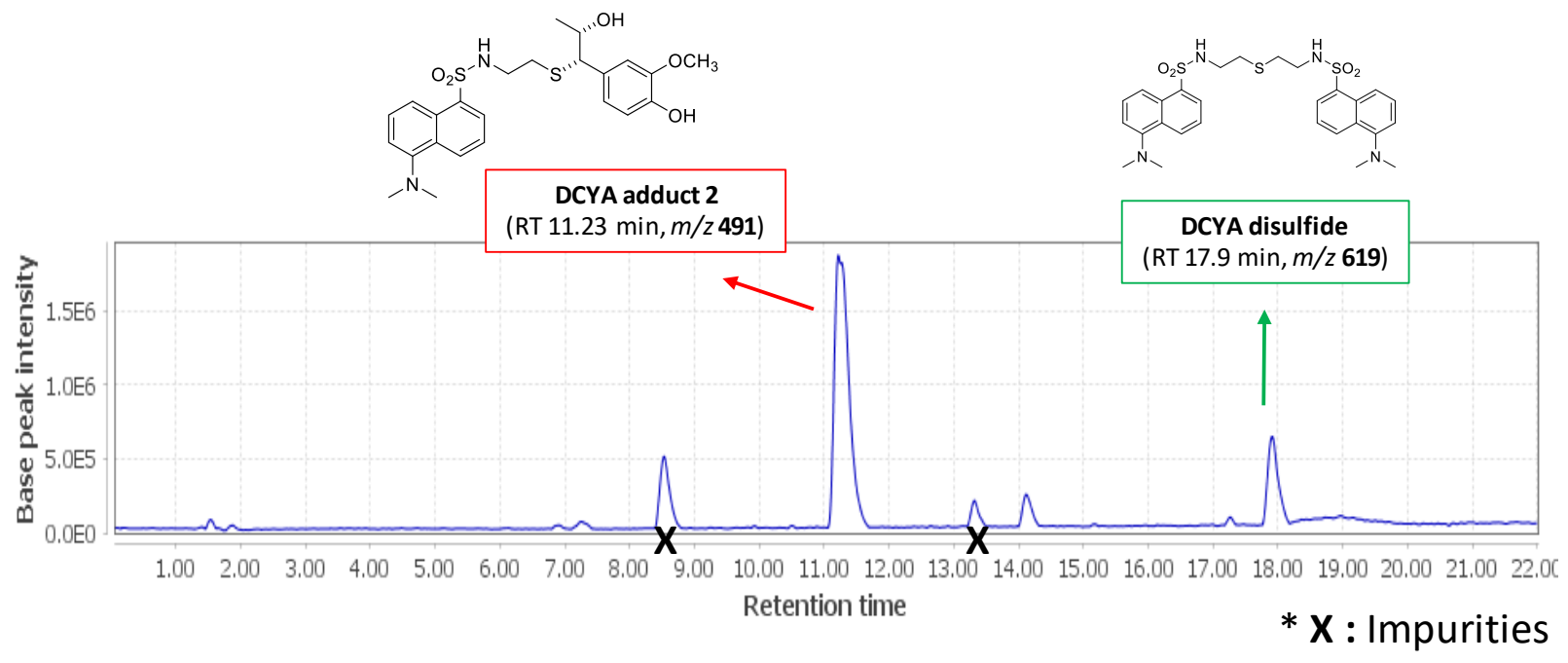


Figure S3. The MS spectrum of DCYA adduct 2 (low resolution, ESI+)

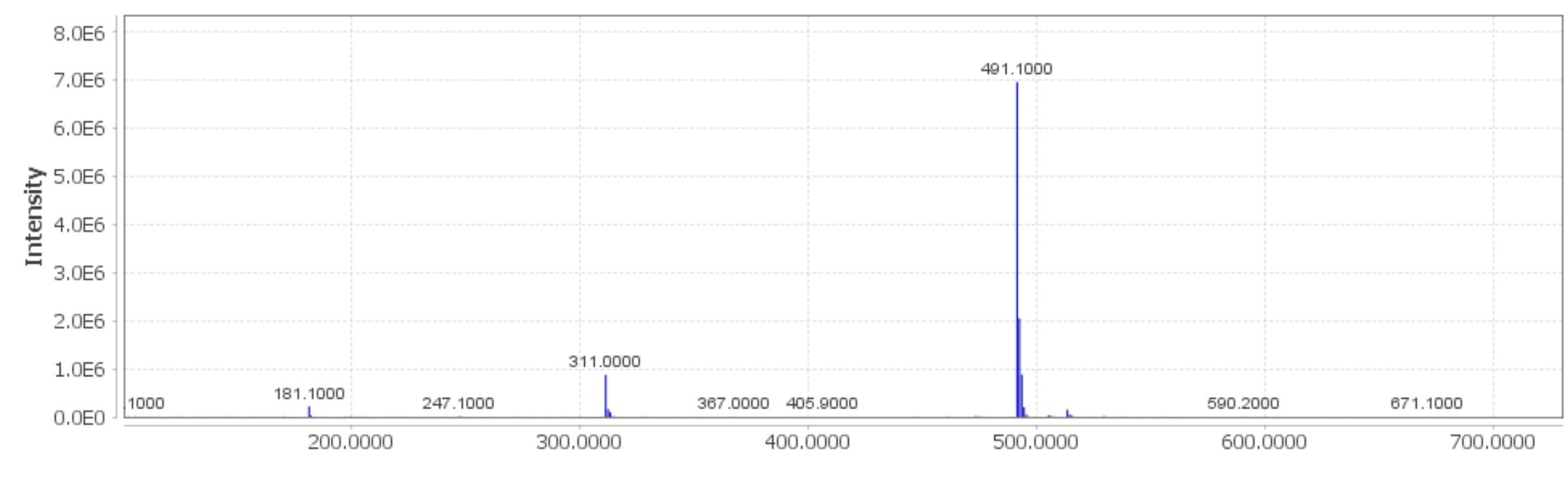

$\mathrm{m} / \mathrm{z}$ 
Table S1. ${ }^{1} \mathrm{H}$ and ${ }^{13} \mathrm{C}$ assignment of isolated DCYA adducts $\mathbf{2}$ and $\mathbf{3}$ in $\mathrm{CDCl}_{3}$.

\begin{tabular}{|c|c|c|c|c|c|}
\hline \multicolumn{3}{|c|}{ Major Adduct (2) } & \multicolumn{3}{|c|}{ Minor Adduct (3) } \\
\hline Position & $\delta_{\mathbf{C}}$ & $\delta_{\mathbf{H}}(J$ in $\mathbf{H z})$ & Position & $\delta_{\mathbf{C}}$ & $\delta_{H}(J$ in $\mathbf{H z})$ \\
\hline 1 & 134.7 & - & $1(x 2)$ & 134.8 & - \\
\hline 2 & 129.7 & $8.17 \mathrm{~d}(7.2)$ & $2(\times 2)$ & 129.7 & $8.20 \mathrm{~d}(7.2)$ \\
\hline 3 & 123.3 & $7.50 \mathrm{dd}(8.5,7.2)$ & $3(x 2)$ & 123.3 & $7.49 \mathrm{dd}(8.5,7.2)$ \\
\hline 4 & 130.7 & $8.54 \mathrm{~d}(8.5)$ & $4(\times 2)$ & 130.7 & $8.53 \mathrm{~d}(8.5)$ \\
\hline 5 & 152.2 & - & $5(x 2)$ & 152.2 & - \\
\hline 6 & 115.4 & $7.19 \mathrm{~d}(7.6)$ & $6(\times 2)$ & 115.4 & $7.18 \mathrm{~d}(7.6)$ \\
\hline 7 & 128.6 & $7.56 \mathrm{dd}(8.6,7.6)$ & $7(x 2)$ & 128.7 & $7.54 \mathrm{dd}(8.6,7.6)$ \\
\hline 8 & 118.8 & $8.24 \mathrm{~d}(8.6)$ & $8(x 2)$ & 118.8 & $8.27 \mathrm{~d}(8.6)$ \\
\hline 9 & 129.5 & - & $9(\mathrm{x} 2)$ & 129.7 & - \\
\hline 10 & 130.0 & - & $10(x 2)$ & 130.0 & - \\
\hline $\mathbf{1}^{\prime}$ & 42.4 & $\begin{array}{l}2.98 \mathrm{dd}(13.1,6.4) \\
2.89 \mathrm{~m}\end{array}$ & $1^{\prime}(x 2)$ & 42.7 & $3.01 \mathrm{~m}$ \\
\hline 2 & 31.5 & $\begin{array}{l}2.44 \mathrm{~m} \\
2.39 \mathrm{~m}\end{array}$ & $2^{\prime}(\mathrm{x} 2)$ & 32.9 & $\begin{array}{l}2.59 \mathrm{~m} \\
2.48 \mathrm{~m}\end{array}$ \\
\hline 1" & 131.4 & - & $1 "$ & 131.1 & - \\
\hline $2 "$ & 110.4 & $6.70 \mathrm{~d}(1.9)$ & $2 "$ & 114.3 & $6.74 \mathrm{~d}(8.1)$ \\
\hline 3" & 146.9 & - & $3^{\prime \prime}$ & 146.9 & - \\
\hline 4" & 145.2 & - & $4^{\prime \prime}$ & 145.8 & - \\
\hline 5" & 114.4 & $6.76 \mathrm{~d}(8.1)$ & $5^{\prime \prime}$ & 109.9 & 6.87 overlap \\
\hline 6" & 121.2 & $6.51 \mathrm{dd}(8.1,1.9)$ & $6^{\prime \prime}$ & 120.8 & $6.62 \mathrm{dd}(8.2,2.0)$ \\
\hline 7" & 59.2 & $3.36 \mathrm{~d}(8.4)$ & $7^{\prime \prime}$ & 52.9 & $4.67 \mathrm{~s}$ \\
\hline 8" & 71.0 & $3.83 \mathrm{~m}$ & & & \\
\hline 9" & 21.0 & $0.98 \mathrm{~d}(6.2)$ & & & \\
\hline $\mathbf{N}\left(\mathrm{CH}_{3}\right)_{2}$ & 45.5 & $2.89 \mathrm{~s}$ & $\mathbf{N}\left(\mathrm{CH}_{3}\right)_{2}$ & 45.6 & $2.88 \mathrm{~s}$ \\
\hline $\mathrm{OCH}_{3}$ & 56.1 & $3.84 \mathrm{~s}$ & $\mathrm{OCH}_{3}$ & 56.2 & $3.86 \mathrm{~s}$ \\
\hline $\mathrm{SO}_{2} \mathrm{NHCH}_{2}$ & & $5.30 \mathrm{t}(6.1)$ & $\mathrm{SO}_{2} \mathrm{NHCH}$ & & $5.32 \mathrm{t}(6.2)$ \\
\hline
\end{tabular}


Table S2. ${ }^{1} \mathrm{H}$ and ${ }^{13} \mathrm{C}$ assignment of 7,4'-oxyneolignans $4 \mathbf{a}$ and $\mathbf{4 b}$

\begin{tabular}{|c|c|c|c|c|c|}
\hline \multicolumn{3}{|c|}{$\begin{array}{l}\text { threo-7,4'-oxyneolignan } \mathbf{4 a} \\
\text { (in Acetone- } d_{6} \text { ) }\end{array}$} & \multicolumn{3}{|c|}{$\begin{array}{c}\text { erythro-7,4'-oxyneolignan } \mathbf{4 b} \\
\left(\text { in }^{-} \mathrm{CDCl}_{3}\right)\end{array}$} \\
\hline Position & $\delta \mathrm{C}$ & $\delta_{H}(J$ in $\mathrm{Hz})$ & Position & $\delta_{\mathbf{C}}$ & $\delta_{H}(J$ in $\mathrm{Hz})$ \\
\hline 1 & 130.8 & - & 1 & 130.2 & - \\
\hline 2 & 111.2 & $7.04 \mathrm{~d}(1.9)$ & 2 & 109.5 & $6.97 \mathrm{~d}(1.5)$ \\
\hline 3 & 148.0 & - & 3 & 146.9 & - \\
\hline 4 & 146.9 & - & 4 & 145.5 & - \\
\hline 5 & 115.1 & $6.78 \mathrm{~d}(8.1)$ & 5 & 114.2 & 6.87 overlap \\
\hline 6 & 120.8 & $6.85 \mathrm{dd}(8.1,1.9)$ & 6 & 120.5 & 6.87 overlap \\
\hline $7^{a}$ & 88.4 & $4.75 \mathrm{~d}(7.4)$ & 7 & 87.2 & $4.86 \mathrm{~d}(4.5)$ \\
\hline $\mathbf{8}^{b}$ & 71.3 & $4.05 \mathrm{~m}$ & 8 & 70.8 & $4.07 \mathrm{~m}$ \\
\hline 9 & 18.4 & $0.99 \mathrm{~d}(6.4)$ & 9 & 17.9 & $1.21 \mathrm{~d}(6.4)$ \\
\hline $1^{\prime}$ & 132.6 & - & 1' & 133.0 & - \\
\hline 2 & 109.9 & $6.98 \mathrm{~d}(1.9)$ & $2^{\prime}$ & 118.2 & 6.70 overlap \\
\hline $3^{\prime}$ & 150.9 & - & $\mathbf{3}^{\prime}$ & 150.6 & - \\
\hline $4^{\prime}$ & 147.5 & - & $4^{\prime}$ & 146.8 & - \\
\hline $5^{\prime}$ & 117.7 & $6.73 \mathrm{~d}(8.3)$ & $5^{\prime}$ & 118.9 & 6.70 overlap \\
\hline $6^{\prime}$ & 118.9 & $6.69 \mathrm{dd}(8.3,1.9)$ & $6^{\prime}$ & 109.4 & 6.87 overlap \\
\hline 7' & 131.2 & $6.27 \mathrm{dd}(15.7,1.6)$ & $7^{\prime}$ & 130.6 & $6.28 \mathrm{dd}(15.7,1.6)$ \\
\hline $8^{\prime}$ & 124.0 & $6.10 \mathrm{dq}(15.7,6.6)$ & $8^{\prime}$ & 124.7 & $6.08 \mathrm{dd}(15.7,6.6)$ \\
\hline 9' & 18.0 & $1.78 \mathrm{dd}(6.6,1.6)$ & $9^{\prime}$ & 18.5 & $1.84 \mathrm{dd}(6.6,1.6)$ \\
\hline $3-\mathrm{OCH}_{3}$ & 55.9 & $3.82 \mathrm{~s}$ & $3-\mathrm{OCH}_{3}$ & 56.1 & $3.90 \mathrm{~s}$ \\
\hline $3^{\prime}-\mathrm{OCH}_{3}$ & 55.9 & $3.88 \mathrm{~s}$ & $3^{\prime}-\mathrm{OCH}_{3}$ & 56.1 & $3.86 \mathrm{~s}$ \\
\hline
\end{tabular}

${ }^{\mathrm{a}} 90.9(\mathrm{C} 7)$ and $4.45 \mathrm{ppm}(\mathrm{H} 7)$ in $\mathrm{CDCl}_{3}{ }^{b} 71.6(\mathrm{C8})$ and $4.09 \mathrm{ppm}(\mathrm{H8})$ in $\mathrm{CDCl}_{3}$ 
Figure S4. The ${ }^{1} \mathrm{H}$ NMR spectrum of $2\left(500 \mathrm{MHz}, \mathrm{CDCl}_{3}\right)$

บับ

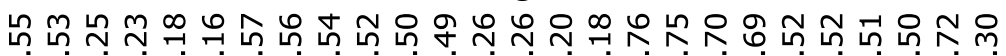

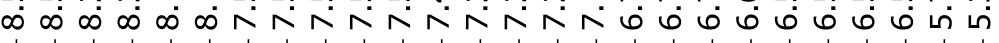

m̊n

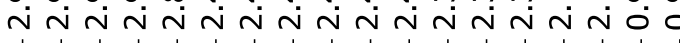
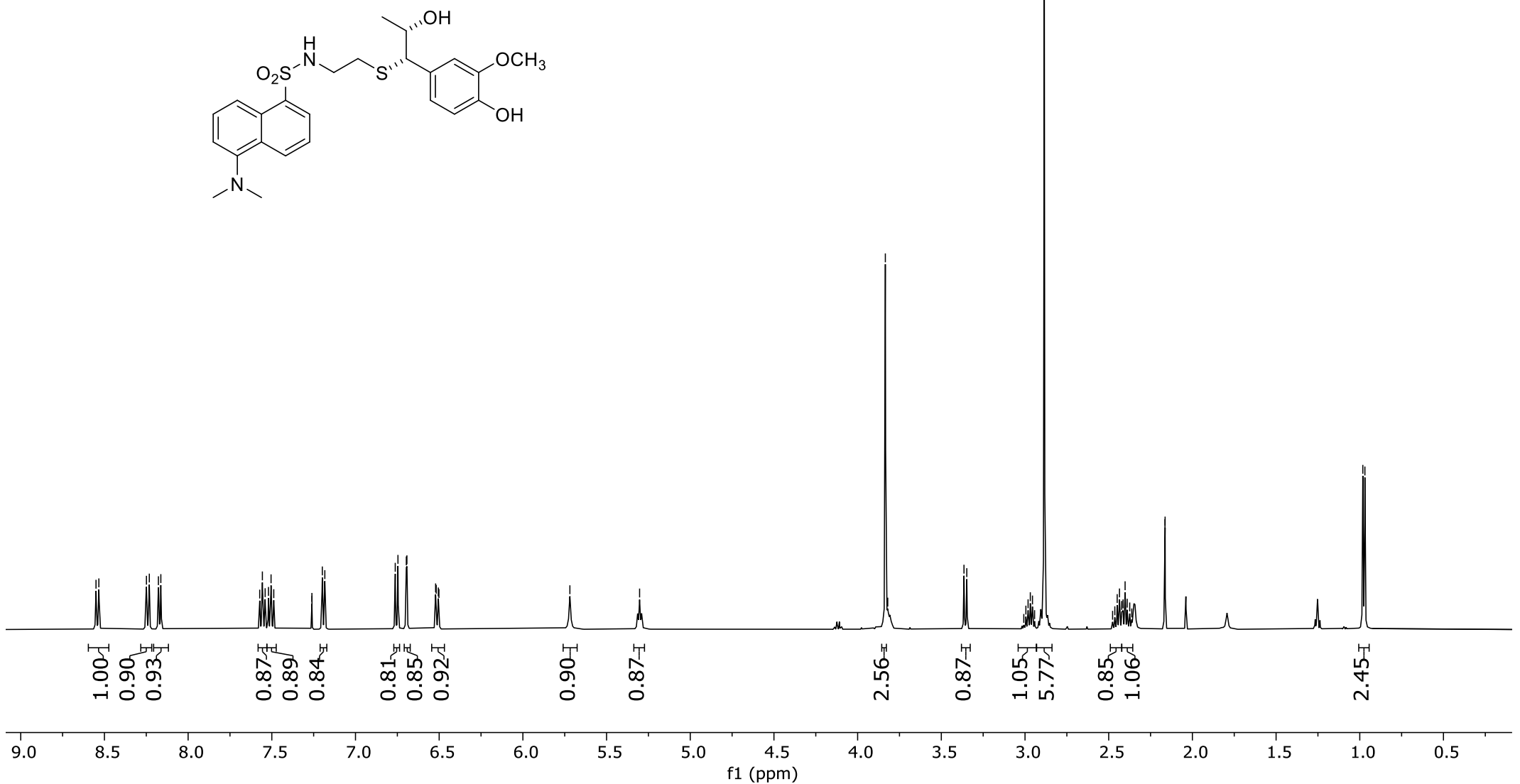
Figure S5. The ${ }^{13} \mathrm{C}$ NMR spectrum of $2\left(125 \mathrm{MHz}, \mathrm{CDCl}_{3}\right)$

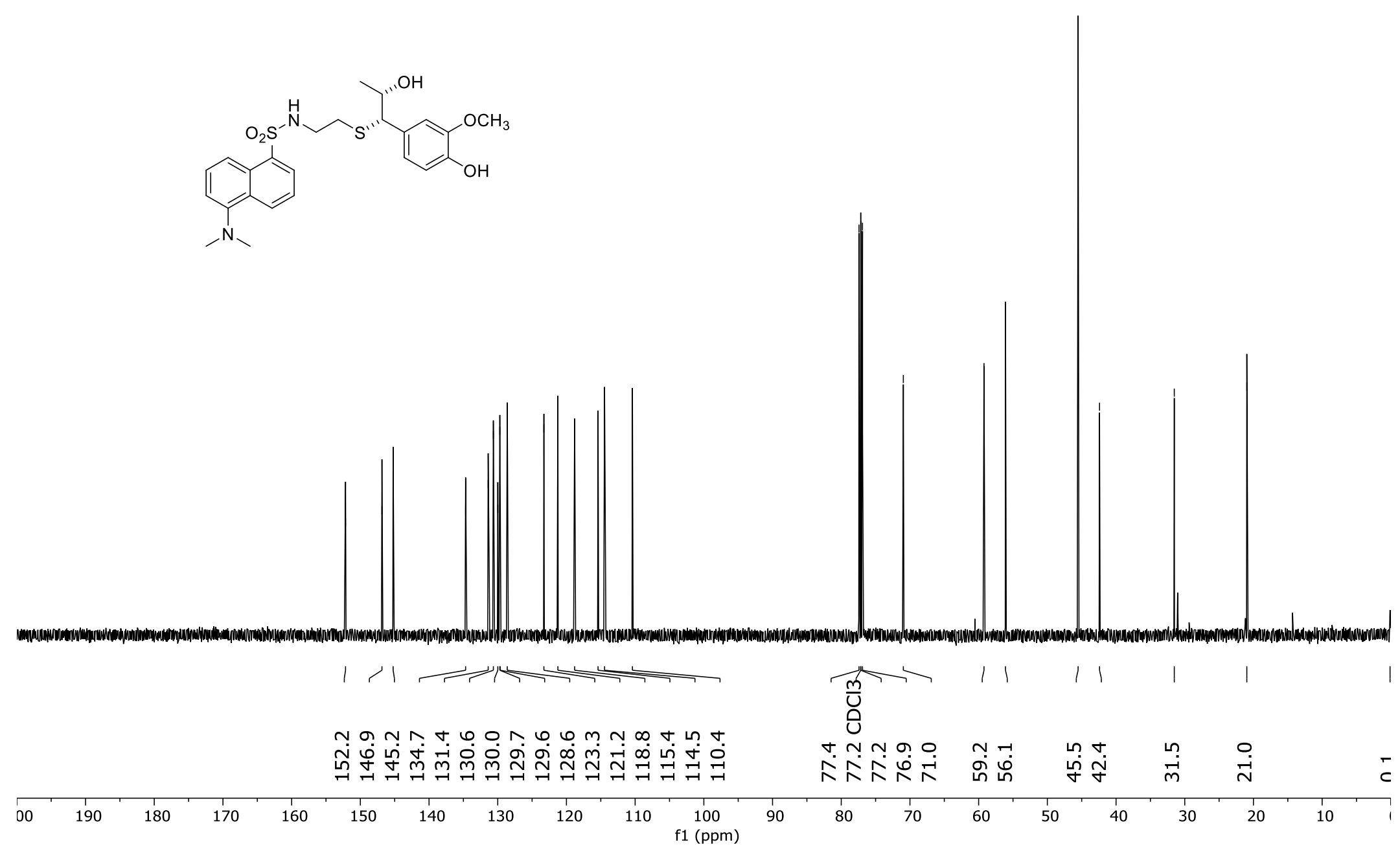


Figure S6. The COSY spectrum of $2\left(500 \mathrm{MHz}, \mathrm{CDCl}_{3}\right)$

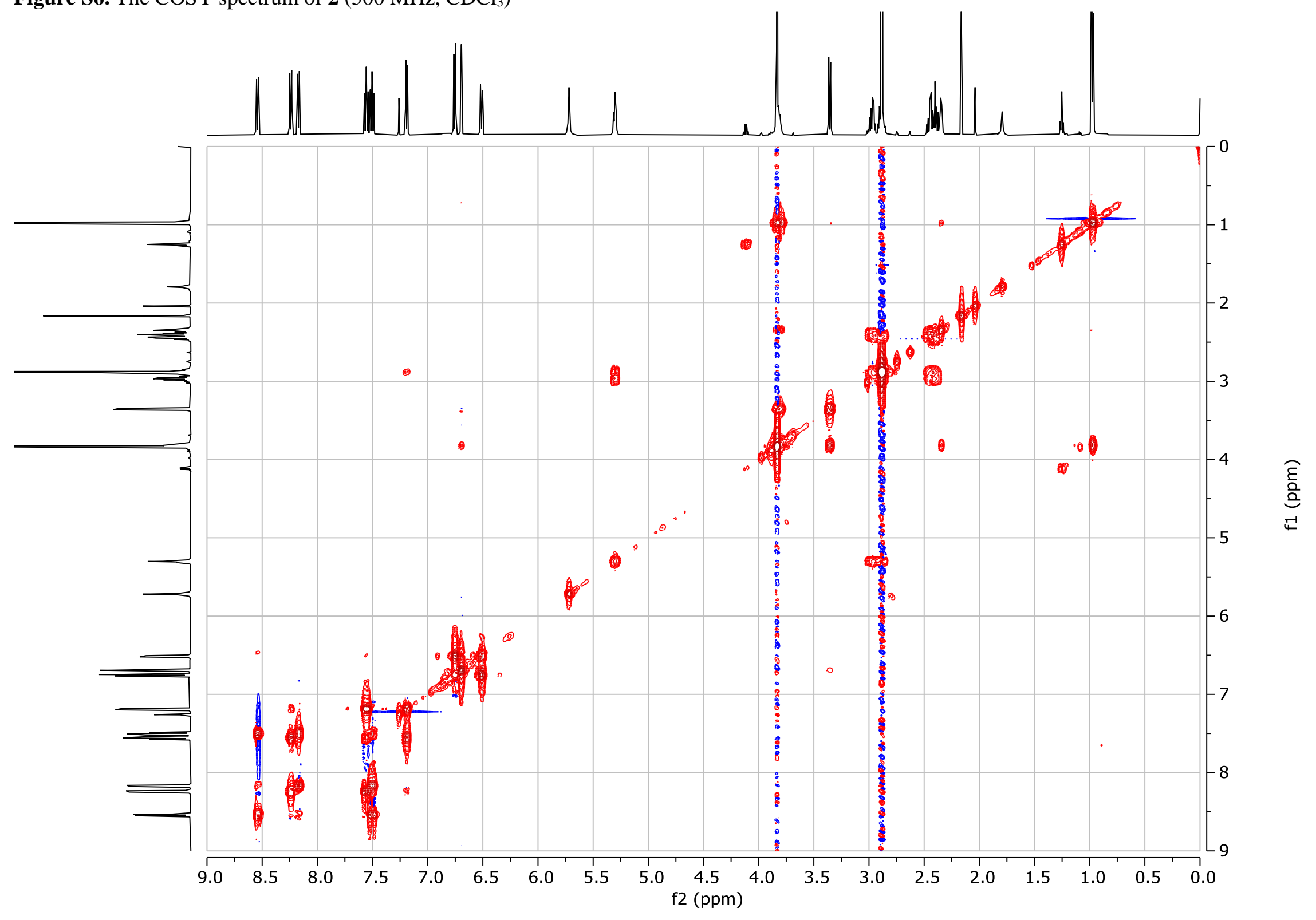


Figure S7. The HSQC spectrum of $2\left(500 \mathrm{MHz}, \mathrm{CDCl}_{3}\right)$

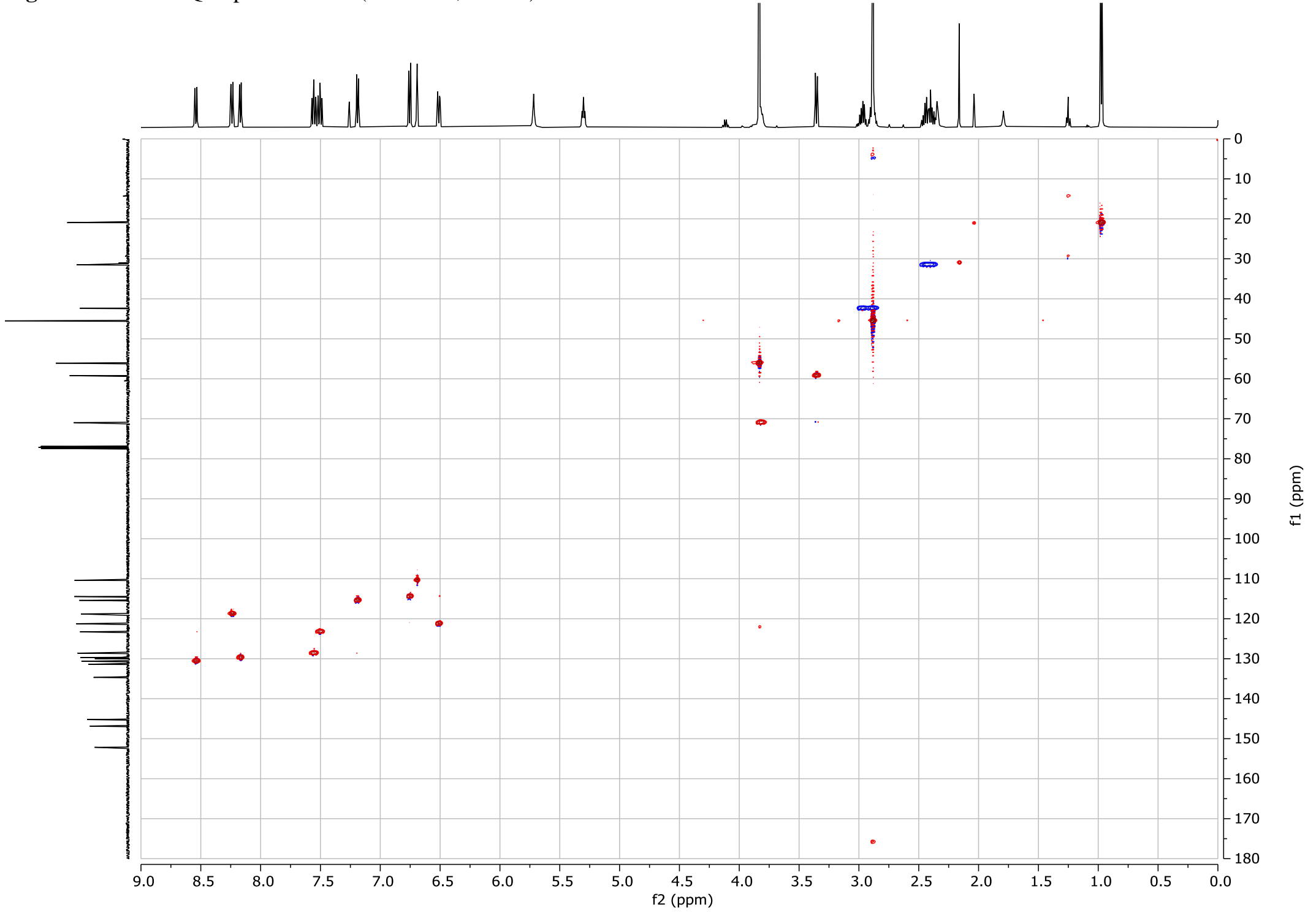


Figure S8. The $\mathrm{HMBC}$ spectrum of $2\left(500 \mathrm{MHz}, \mathrm{CDCl}_{3}\right)$

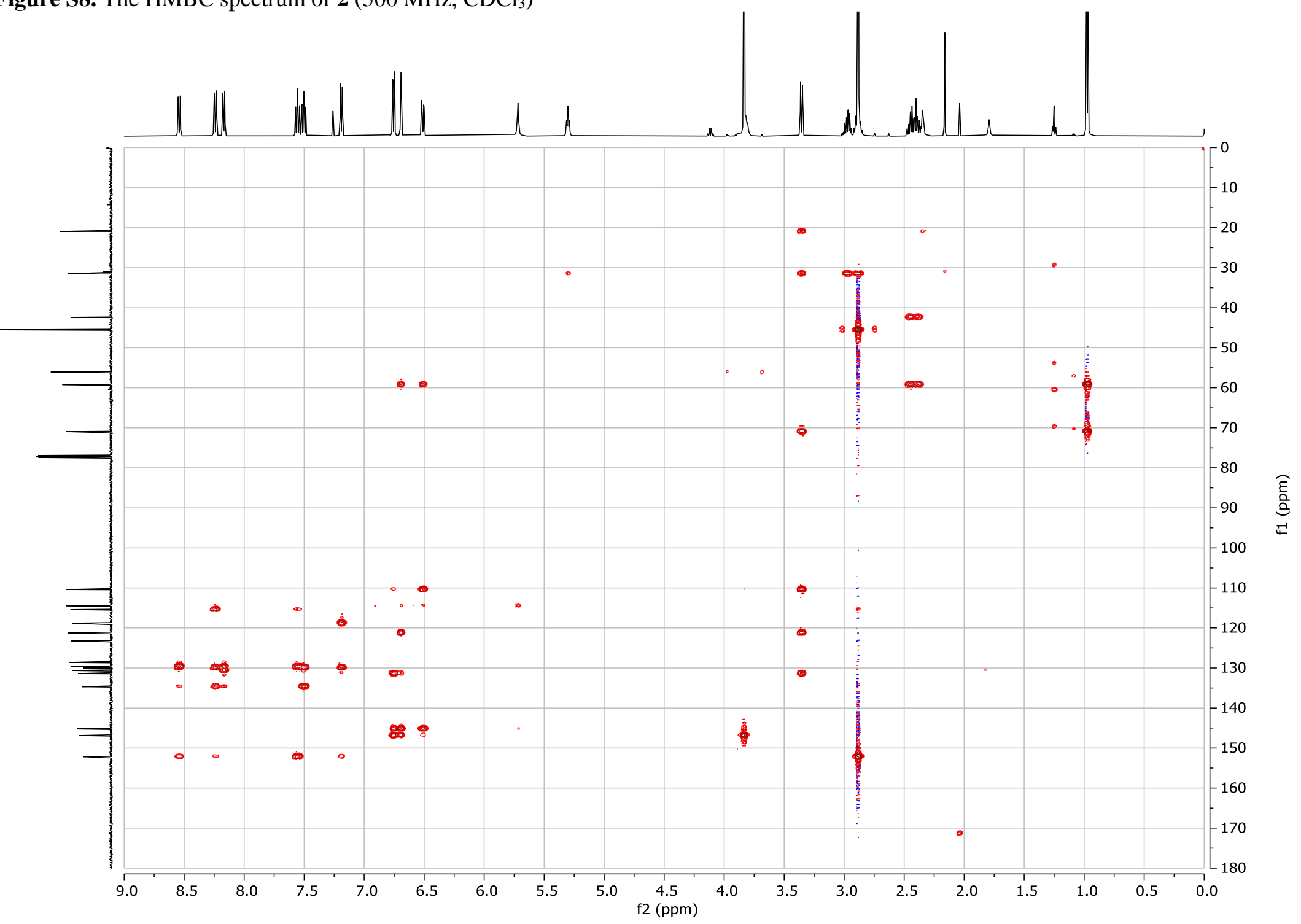


Figure S9. The ${ }^{1} \mathrm{H}$ NMR spectrum of the mixture $\mathbf{3}$ and $\mathbf{4 b}\left(500 \mathrm{MHz}, \mathrm{CDCl}_{3}\right)$

$$
\text { 商 }
$$

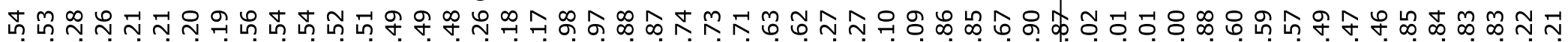
象
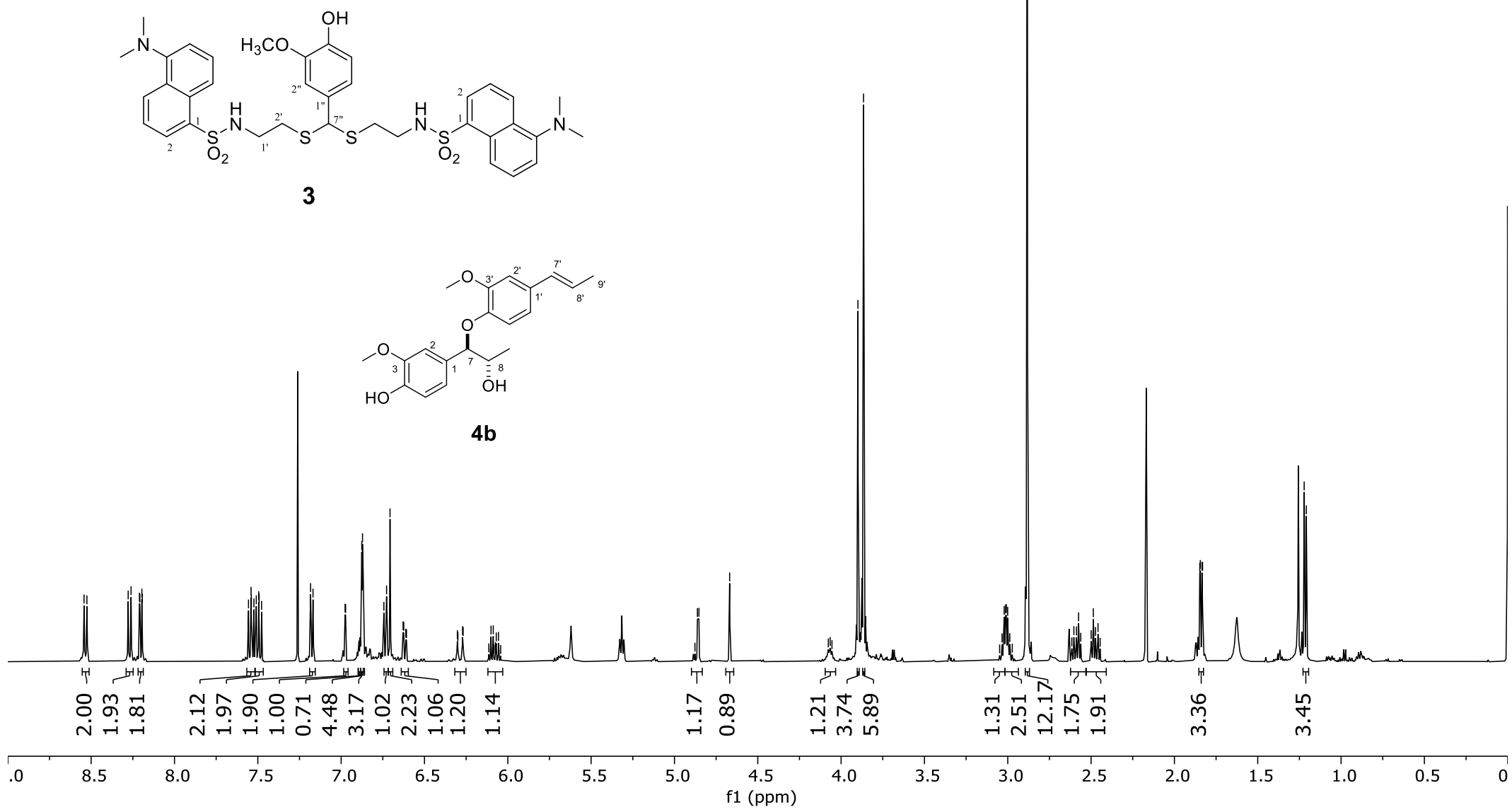
Figure S10. The ${ }^{13} \mathrm{C}$ NMR spectrum of the mixture 3 and $\mathbf{4 b}\left(125 \mathrm{MHz}, \mathrm{CDCl}_{3}\right)$

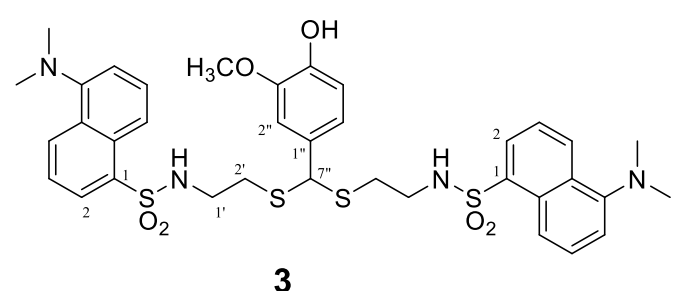

3

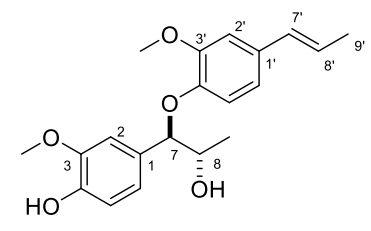

4b
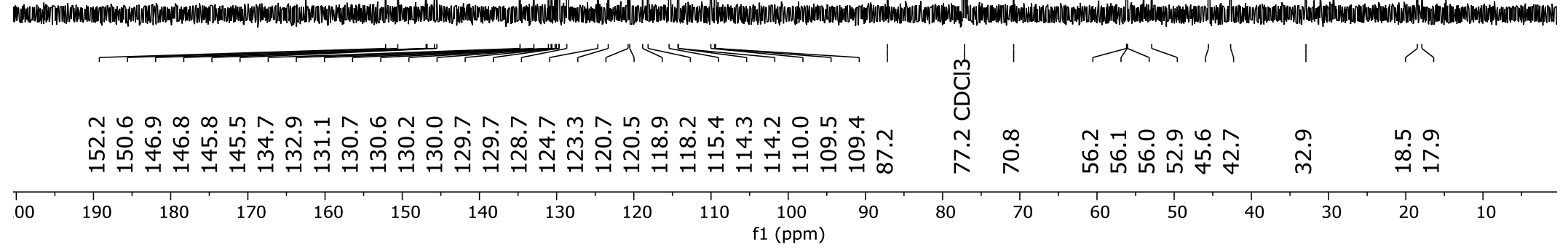
Figure S11. The COSY spectrum of the mixture $\mathbf{3}$ and $\mathbf{4 b}\left(500 \mathrm{MHz}, \mathrm{CDCl}_{3}\right)$

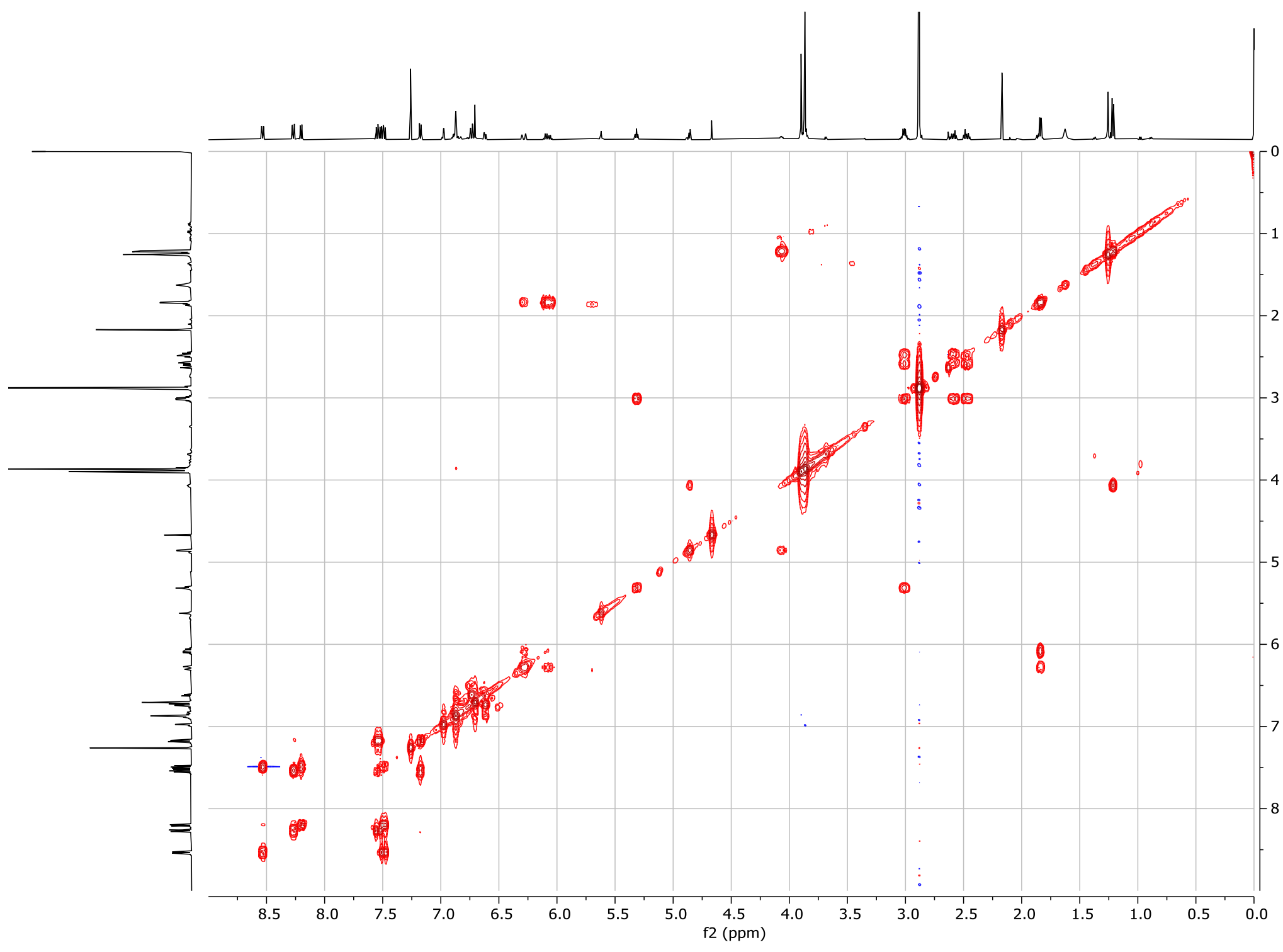


Figure S12. The HSQC spectrum of the mixture $\mathbf{3}$ and $\mathbf{4 b}\left(500 \mathrm{MHz}, \mathrm{CDCl}_{3}\right)$

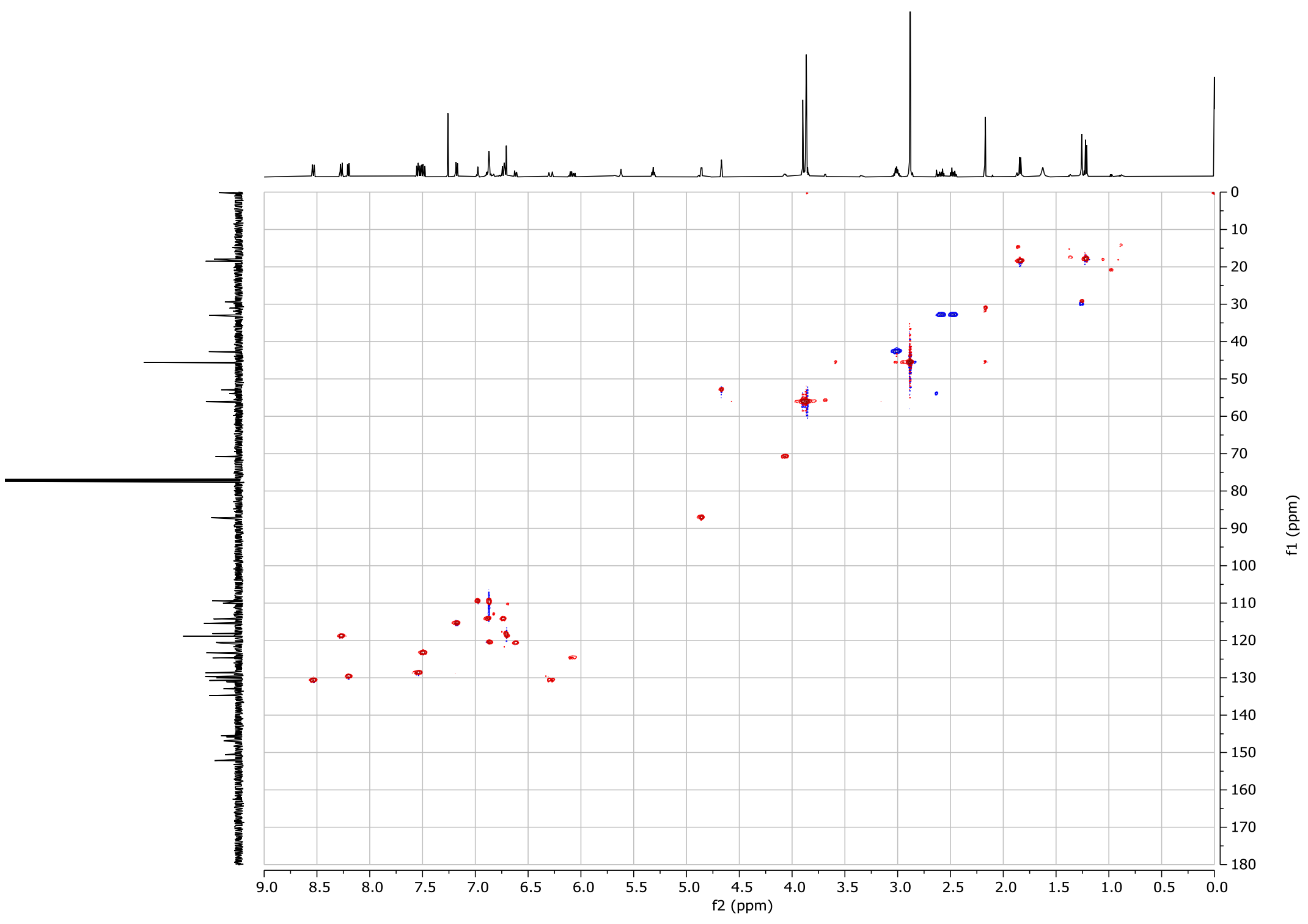


Figure S13. The $\mathrm{HMBC}$ spectrum of the mixture $\mathbf{3}$ and $\mathbf{4 b}\left(500 \mathrm{MHz}, \mathrm{CDCl}_{3}\right)$

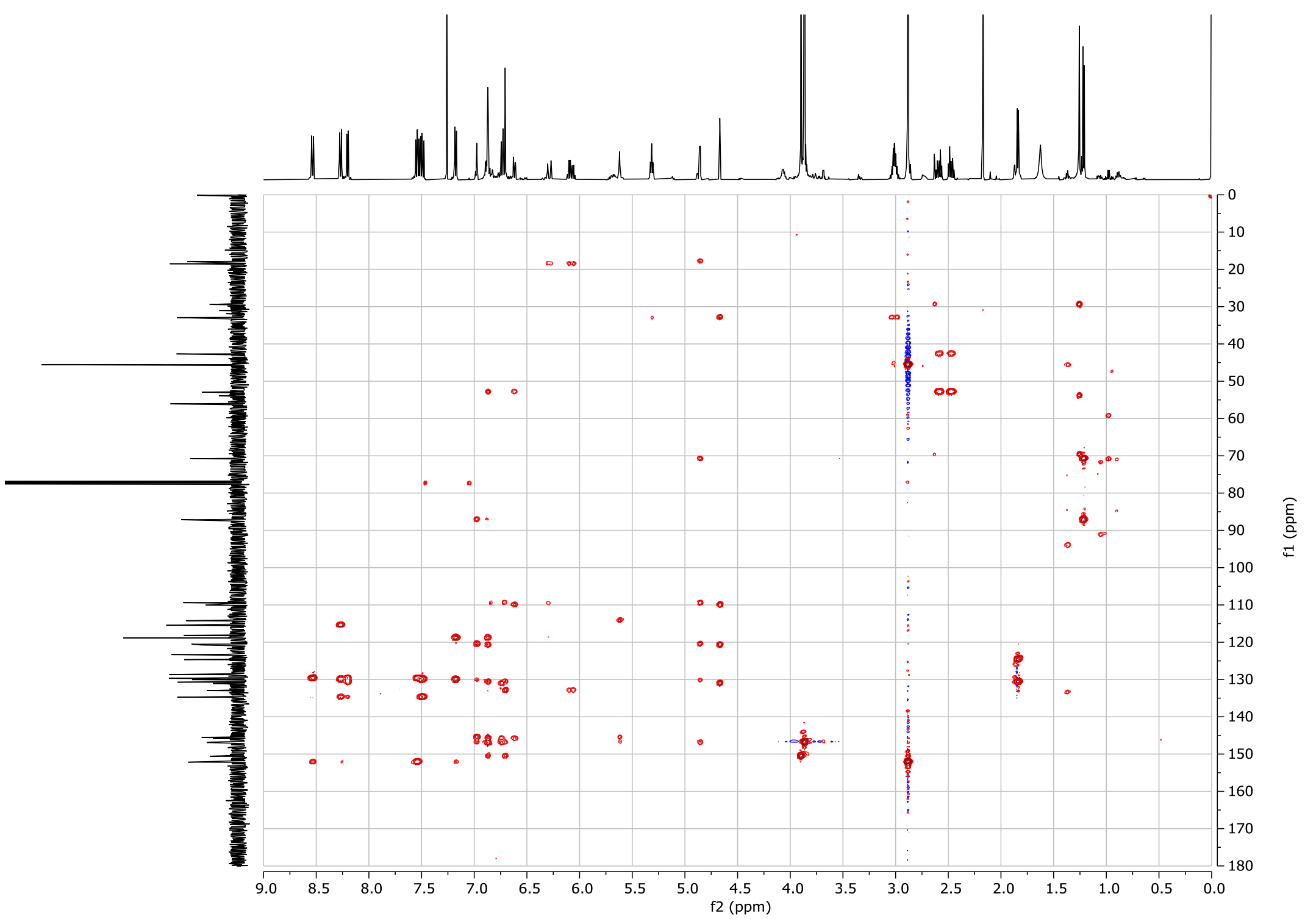


Figure S14. Failed attempts to synthesize isoeugenol epoxide

1) $m$-CPBA

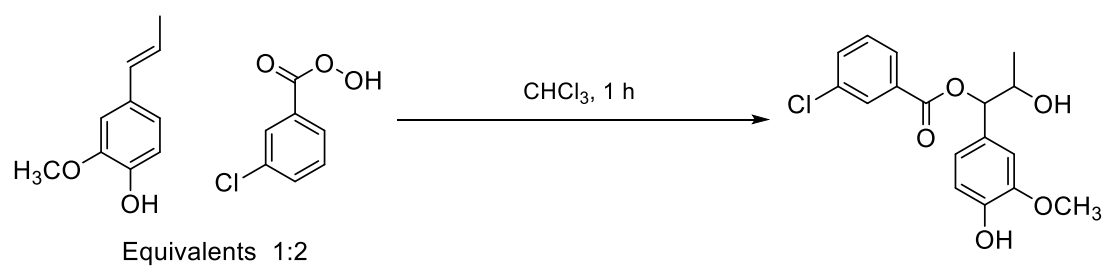

2) $t-\mathrm{BP}$

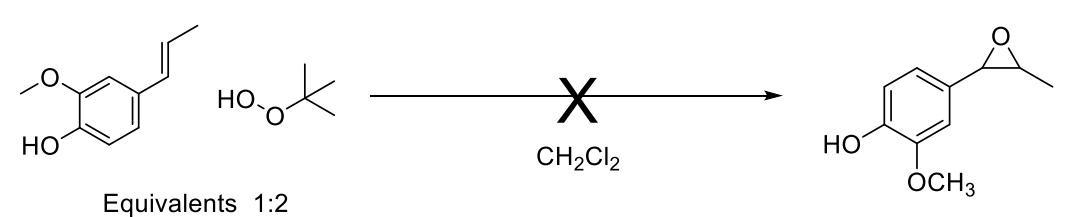

3) 1. $\mathrm{NBS} / 2 . \mathrm{K}_{2} \mathrm{CO}_{3}$
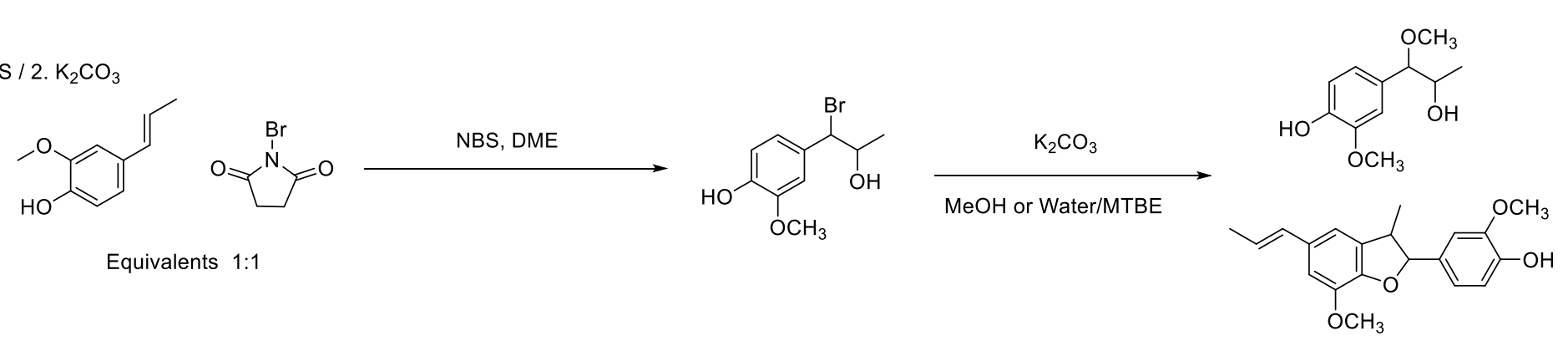

4) 1. Acetylation / 2. m-CPBA / 3. Deacetylation

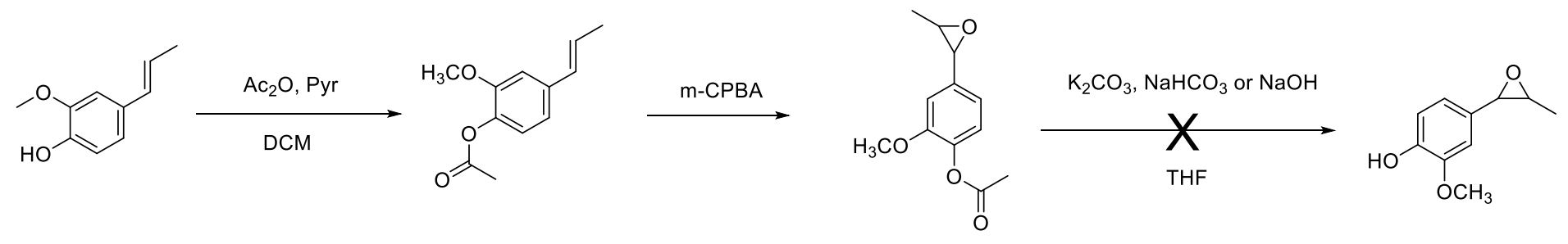


Figure S15. The ${ }^{1} \mathrm{H}$ NMR spectrum of aged isoeugenol mixture $\left(500 \mathrm{MHz}, \mathrm{CDCl}_{3}\right)$. Expansion between $\delta_{\mathrm{H}} 6.5-4.1$ is shown in inset.

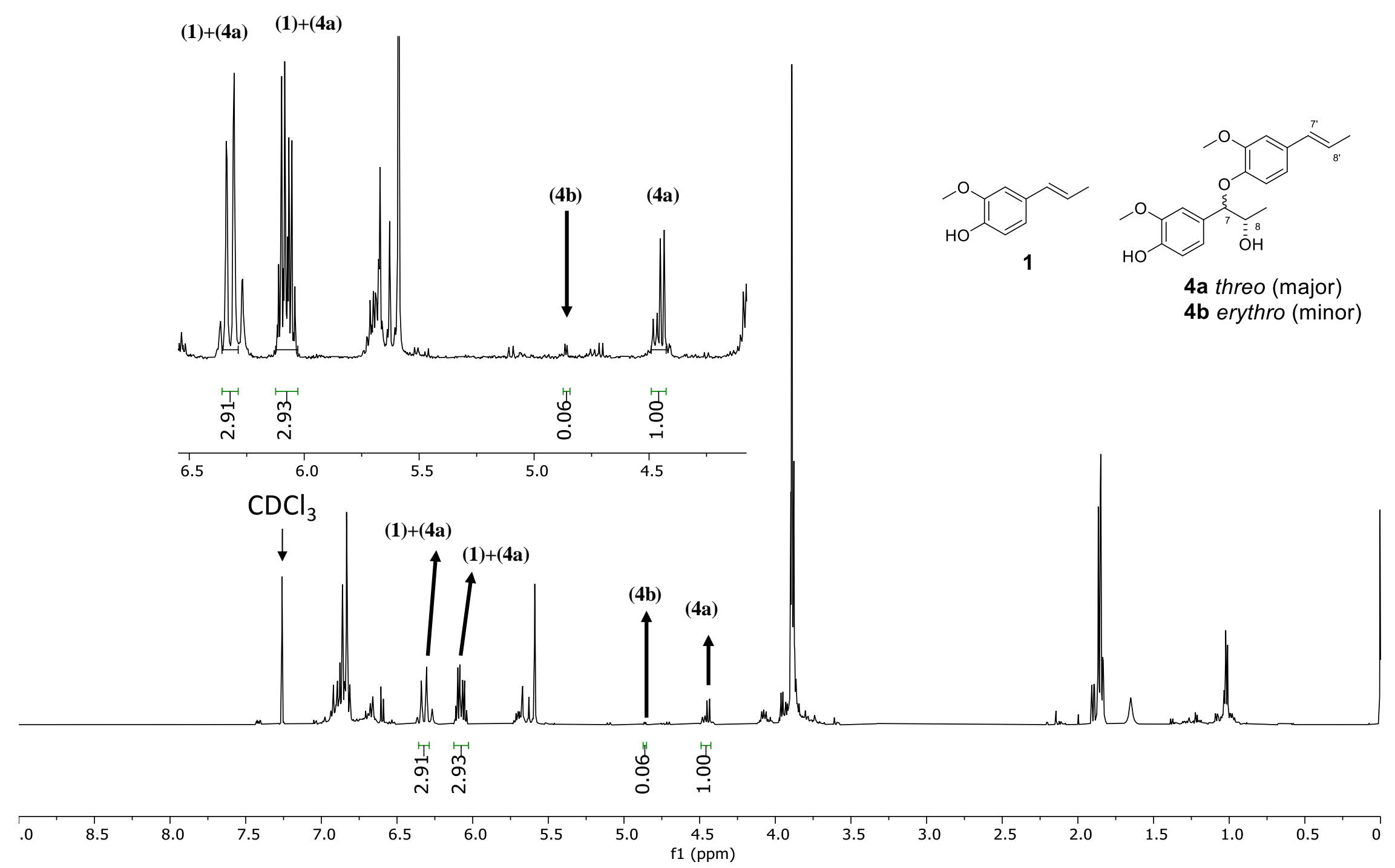


Figure S16. ${ }^{13} \mathrm{C}$ NMR spectrum of aged isoeugenol mixture (125 $\left.\mathrm{MHz}, \mathrm{CDCl}_{3}\right)$

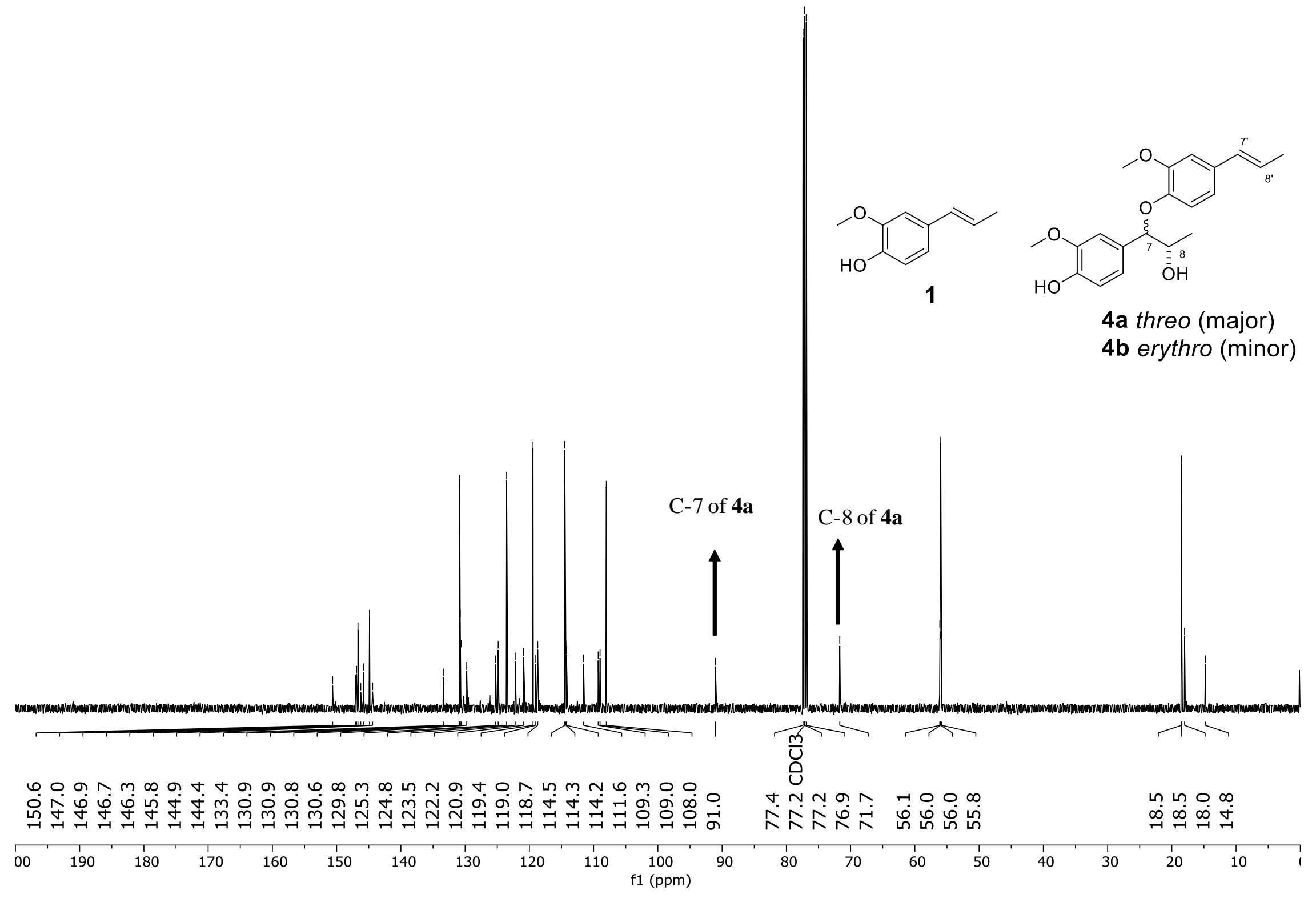


Figure S17. ${ }^{1} \mathrm{H}$ NMR spectrum of $\mathbf{4 a}\left(500 \mathrm{MHz}\right.$, Acetone- $\left.d_{6}\right)$.

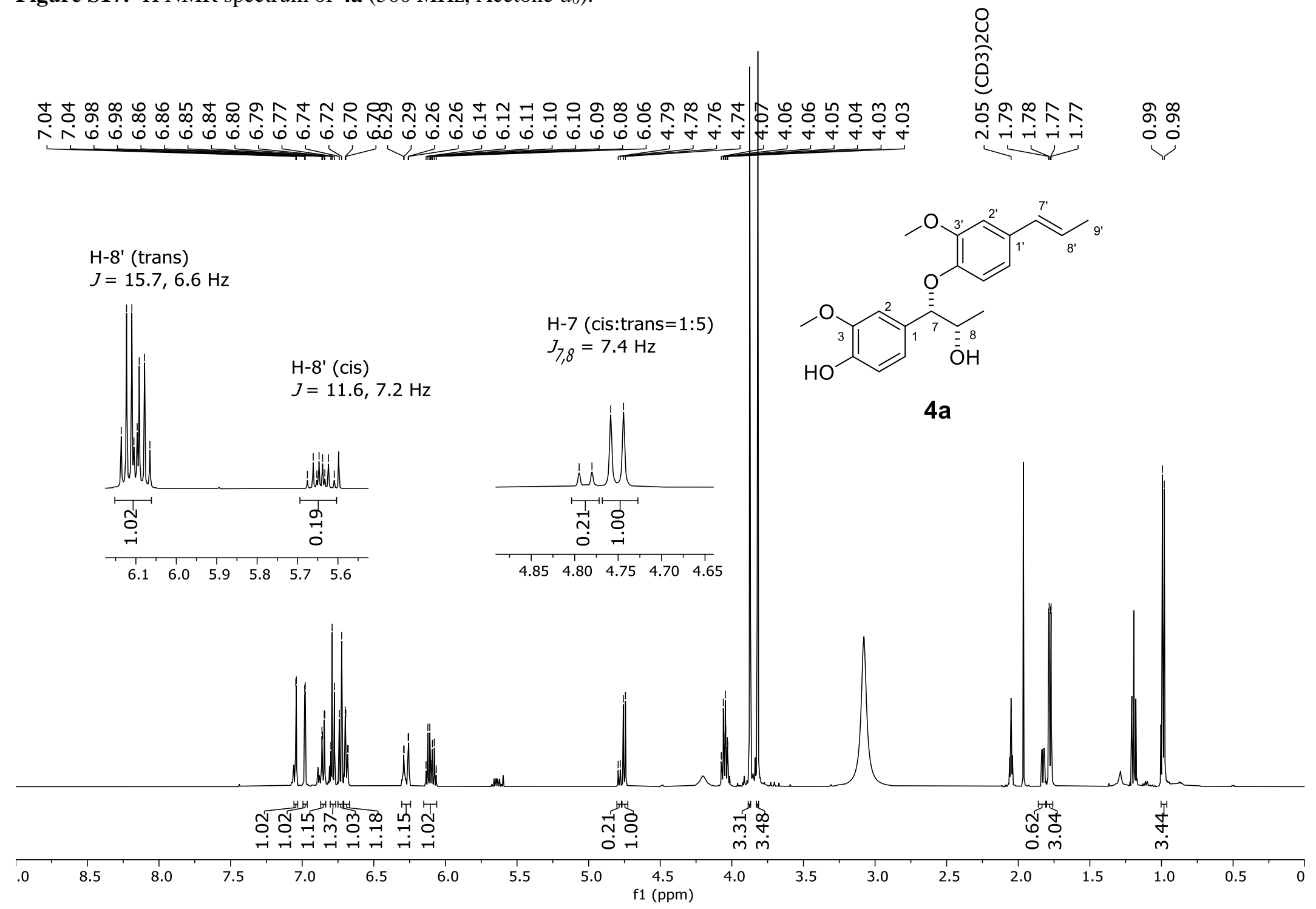


Figure S18. ${ }^{13} \mathrm{C}$ NMR spectrum of $4 \mathbf{a}\left(125 \mathrm{MHz}\right.$, Acetone- $\left.d_{6}\right)$

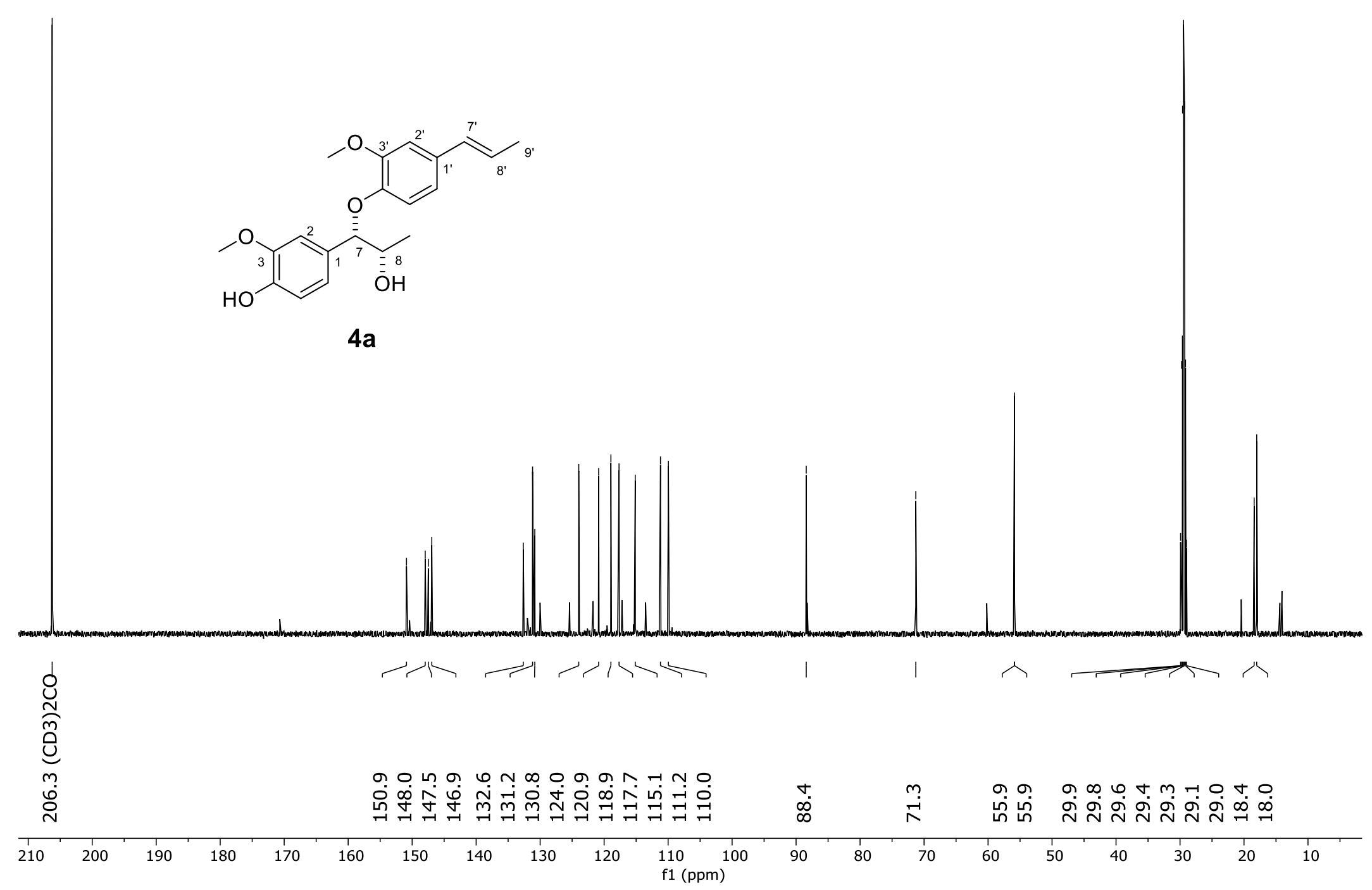


Figure S19. The COSY NMR spectrum of $\mathbf{4 a}\left(500 \mathrm{MHz}\right.$, Acetone- $\left.d_{6}\right)$

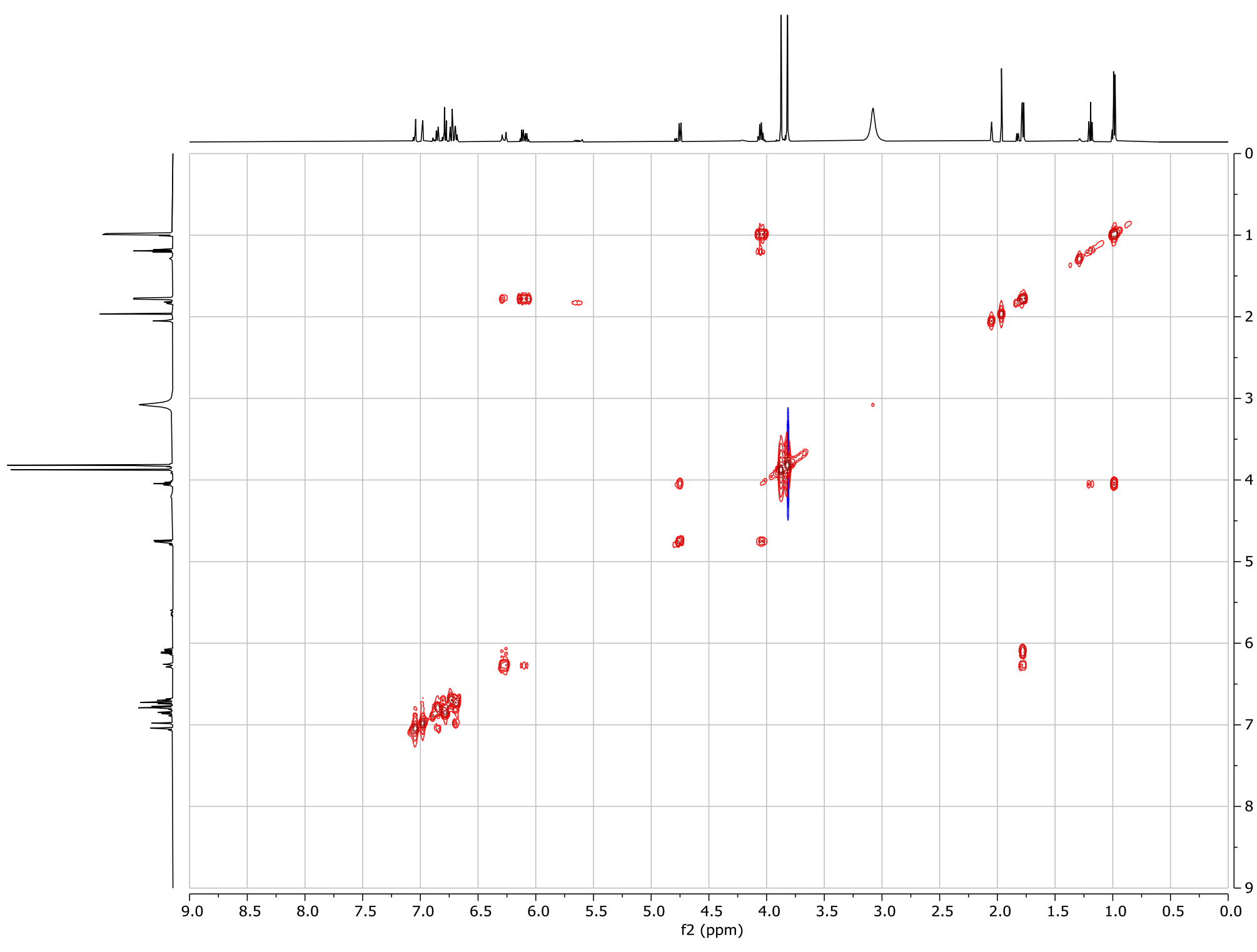


Figure S20. The HSQC NMR spectrum of $\mathbf{4 a}\left(500 \mathrm{MHz}\right.$, Acetone- $\left.d_{6}\right)$

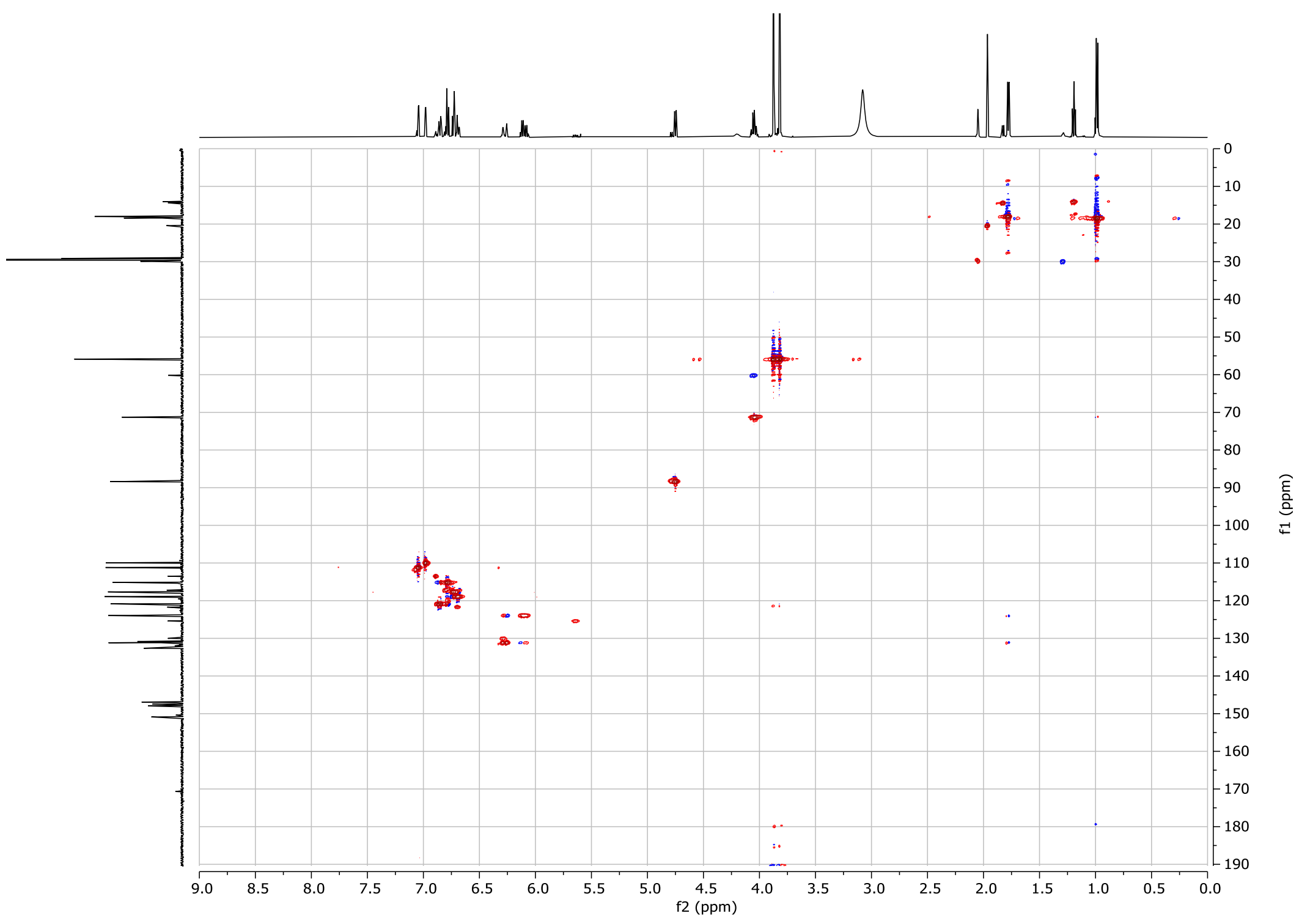


Figure S21. The HMBC NMR spectrum of $\mathbf{4 a}\left(500 \mathrm{MHz}\right.$, Acetone- $\left.d_{6}\right)$

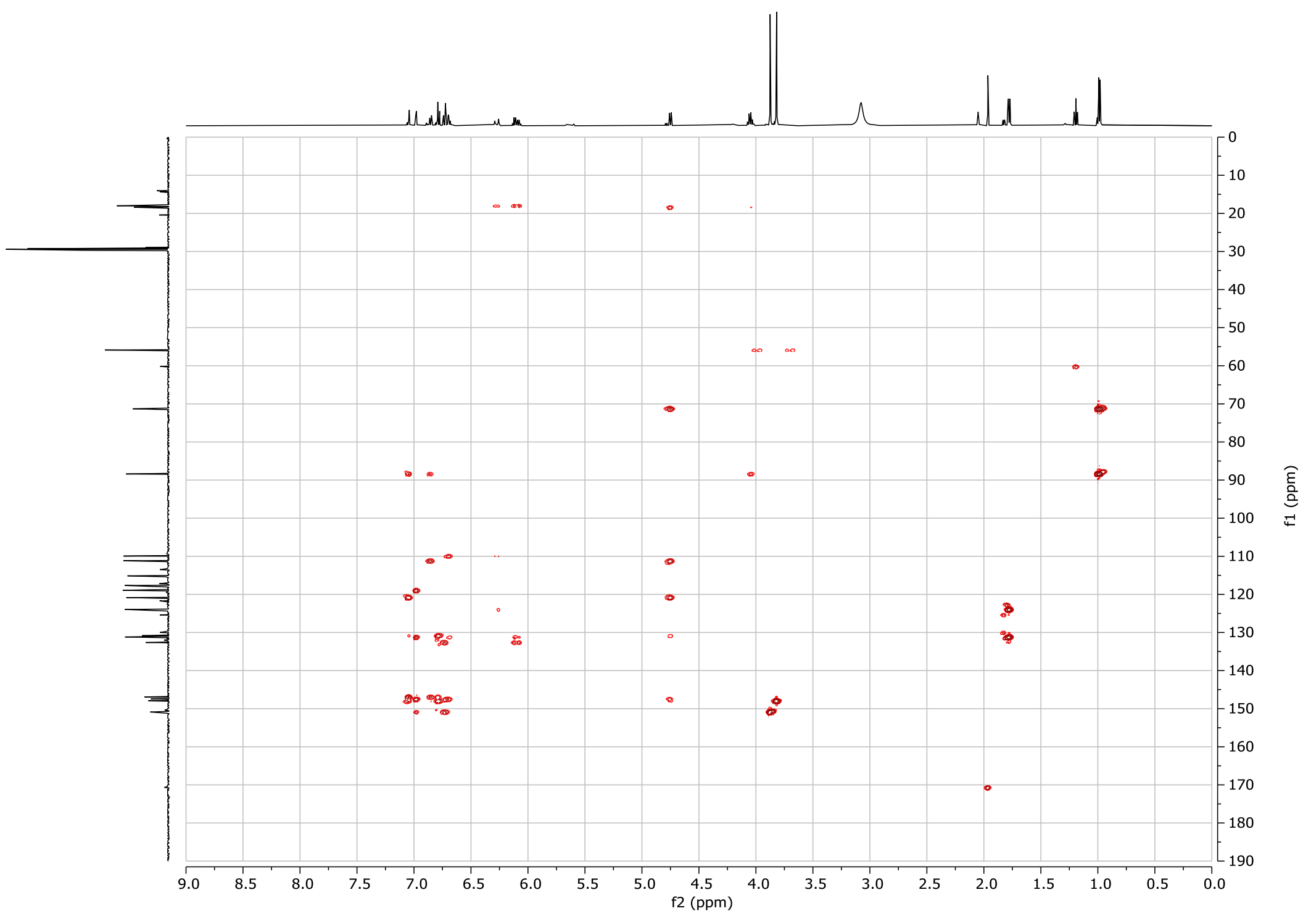


Figure S22. Arrayed ${ }^{1} \mathrm{H}$ NMR spectra for 7,4'-oxyneolignan 4a, DCYA, region of products $(\mathbf{2}, \mathbf{3})$, before addition of $\mathrm{DBN}\left(500 \mathrm{MHz}, \mathrm{CDCl}_{3}\right)$

DCYA

$\mathrm{H}-8\left(\delta_{\mathrm{H}} 8.31 \sim 8.27\right)$

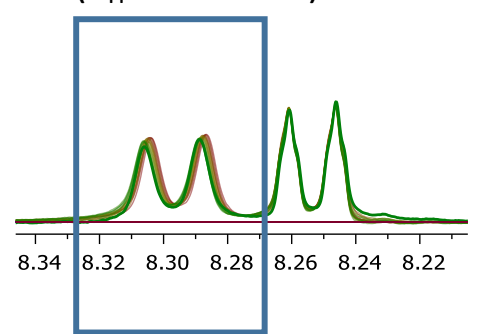

Isomer trace of $\mathbf{4 a}$

at region of Isoeugenol (1)

$\left(\delta_{H} 6.35 \sim 6.31\right)$

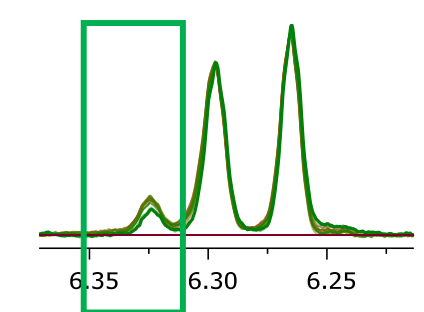

Internal Standard
$\left(\delta_{\mathrm{H}} 5.84^{\sim} 5.81\right)$

DCYA adduct 2

$\mathrm{H}-2\left(\delta_{\mathrm{H}} 8.20 \sim 8.15\right)$
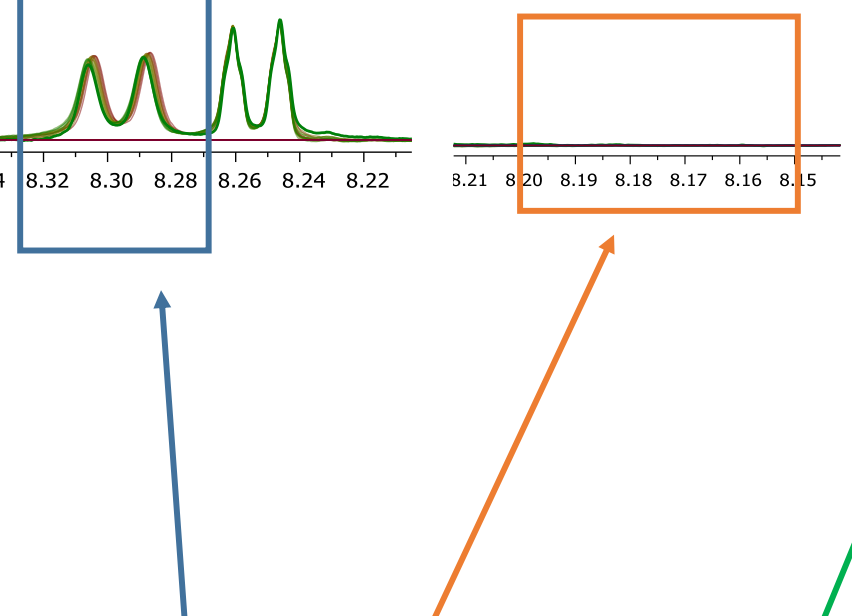

Internal Standard
$\left(\delta_{\mathrm{H}} 5.84 \sim 5.81\right)$
$4 a\left(\delta_{H} 4.50 \sim 4.42\right)$

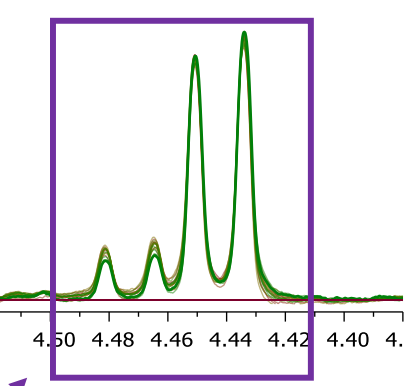

${ }^{1} \mathrm{H} \mathrm{NMR}\left(500 \mathrm{MHz}, \mathrm{CDCl}_{3}\right)$

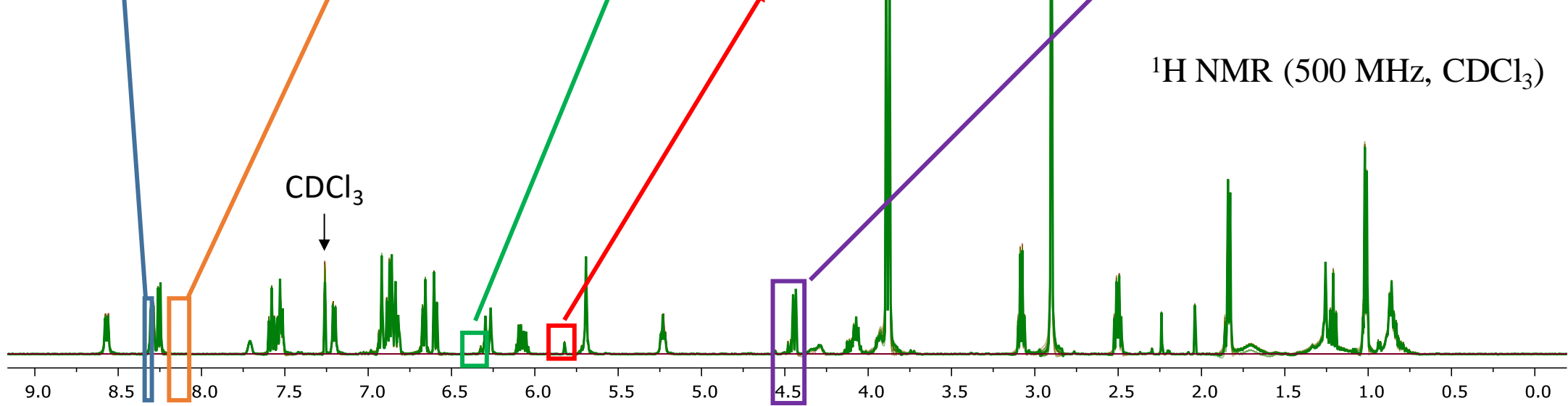


Figure S23. Arrayed ${ }^{1} \mathrm{H}$ NMR spectra for 7,4'-oxyneolignan 4a and DCYA, after addition of DBN (500 MHz, $\left.\mathrm{CDCl}_{3}\right)$

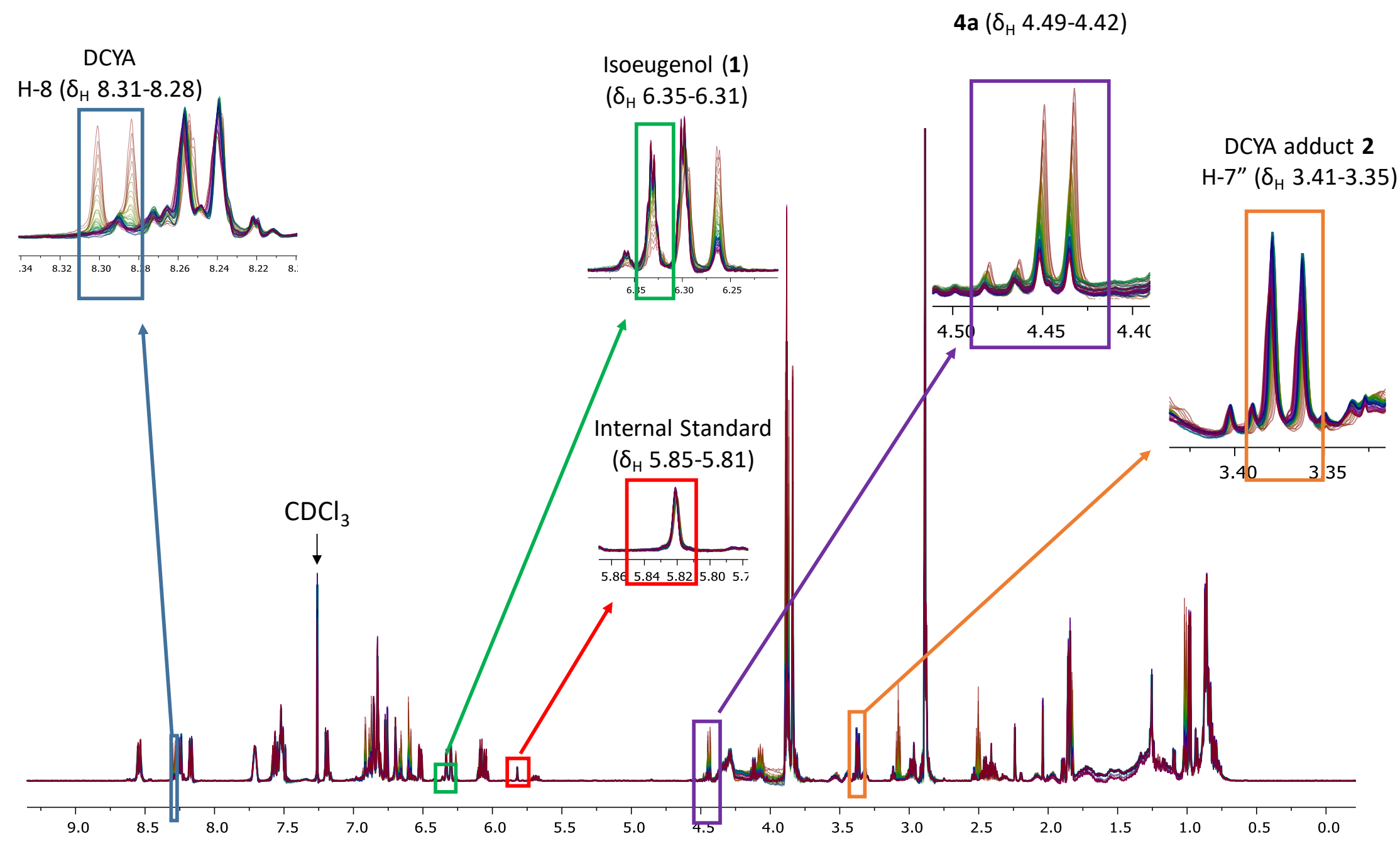


Table S3. Select normalized integral values of DCYA, compound 4a and region of products before addition of DBN (2,5-dimethylfuran was used as internal standard).

\begin{tabular}{|c|c|c|c|c|c|c|c|c|c|c|c|c|}
\hline \multirow[t]{2}{*}{$\begin{array}{l}\text { Time } \\
(\min )\end{array}$} & \multicolumn{2}{|c|}{ 7,4'-oxyneolignan 4a } & \multicolumn{2}{|c|}{ DCYA } & \multicolumn{2}{|c|}{$\begin{array}{l}\text { Isomer trace of } 4 a \text { at region of } \\
\text { Isoeugenol (1) }\end{array}$} & \multicolumn{2}{|c|}{ DCYA adduct 2} & \multicolumn{4}{|c|}{ doEs (Depletion of electrophile signals, \%, average) } \\
\hline & $\begin{array}{c}\mathrm{H}-7 \\
\left(\delta_{\mathrm{H}}^{4.49-4.42)}\right.\end{array}$ & $\begin{array}{c}\mathrm{H}-5 \\
\left(\delta_{\mathrm{H}} 6.70-6.63\right)\end{array}$ & $\begin{array}{c}\mathrm{H}-1^{\prime} \\
\left(\delta_{\mathrm{H}} 3.12-3.05\right)\end{array}$ & $\begin{array}{c}\mathrm{H}-8 \\
\left(\delta_{\mathrm{H}} 8.33-8.27\right)\end{array}$ & $\begin{array}{l}\text { Half peak of H-7 } \\
\left(\delta_{\mathrm{H}} 6.35-6.31\right)\end{array}$ & $\begin{array}{l}\text { Half peak of H-9 } \\
\left(\delta_{\mathrm{H}} 1.87-1.85\right)\end{array}$ & $\begin{array}{l}\text { Integral ratio of } \mathrm{H}-2 \\
\left(\delta_{\mathrm{H}} 8.20-8.15\right)\end{array}$ & $\begin{array}{c}\text { Integral ratio of H-7" } \\
\left(\delta_{\mathrm{H}} 3.41-3.35\right)\end{array}$ & $\begin{array}{c}7,4^{\prime}- \\
\text { oxyneolignan } \\
\mathbf{4 a} \\
\end{array}$ & DCYA & $\begin{array}{c}\text { Region of } \\
\text { DCYA } \\
\text { adduct (2) }\end{array}$ & $\begin{array}{c}\text { Isomer trace of } \\
\text { 4a at region of } \\
\text { Isoeugenol }\end{array}$ \\
\hline 9.0 & 100.00 & 100.00 & 100.00 & 100.00 & 20.67 & 27.36 & -0.02 & 0.77 & 100.00 & 100.00 & 0.37 & 24.01 \\
\hline 14.0 & 107.60 & 112.10 & 105.79 & 109.28 & 22.57 & 24.17 & -0.30 & 1.05 & 109.85 & 107.54 & 0.37 & 23.37 \\
\hline 19.0 & 99.12 & 100.89 & 96.42 & 101.91 & 23.10 & 22.26 & -0.34 & 0.73 & 100.01 & 99.16 & 0.19 & 22.68 \\
\hline 24.0 & 100.58 & 100.43 & 97.52 & 101.45 & 26.21 & 28.31 & -0.41 & 0.80 & 100.50 & 99.48 & 0.20 & 27.26 \\
\hline 29.0 & 99.71 & 103.30 & 97.08 & 101.95 & 25.43 & 21.93 & -0.47 & 0.89 & 101.50 & 99.51 & 0.21 & 23.68 \\
\hline 34.0 & 99.31 & 103.93 & 97.14 & 101.26 & 22.45 & 19.88 & -0.67 & 0.63 & 101.62 & 99.20 & -0.02 & 21.17 \\
\hline 39.0 & 100.69 & 104.34 & 98.46 & 103.18 & 26.35 & 21.75 & -0.53 & 1.21 & 102.52 & 100.82 & 0.34 & 24.05 \\
\hline 44.0 & 98.37 & 102.50 & 96.15 & 98.72 & 18.22 & 17.10 & -0.47 & 0.86 & 100.43 & 97.44 & 0.19 & 17.66 \\
\hline 49.0 & 104.74 & 105.19 & 104.34 & 104.45 & 28.10 & 31.90 & -0.23 & 0.86 & 104.96 & 104.39 & 0.32 & 30.00 \\
\hline 54.0 & 103.21 & 104.94 & 99.40 & 101.60 & 21.42 & 22.09 & -0.23 & 0.96 & 104.07 & 100.50 & 0.37 & 21.76 \\
\hline 59.0 & 102.78 & 106.59 & 99.43 & 103.46 & 23.97 & 20.44 & -0.41 & 1.15 & 104.69 & 101.45 & 0.37 & 22.21 \\
\hline 64.0 & 103.11 & 112.08 & 101.24 & 103.98 & 20.77 & 17.87 & -0.67 & 1.11 & 107.60 & 102.61 & 0.22 & 19.32 \\
\hline 69.0 & 102.59 & 104.89 & 97.32 & 99.18 & 26.92 & 26.42 & -0.94 & 1.33 & 103.74 & 98.25 & 0.19 & 26.67 \\
\hline 74.0 & 104.53 & 109.90 & 100.81 & 103.20 & 25.10 & 18.85 & -0.79 & 1.28 & 107.22 & 102.00 & 0.24 & 21.97 \\
\hline 79.0 & 104.27 & 111.97 & 101.21 & 103.23 & 28.11 & 19.22 & -1.31 & 1.31 & 108.12 & 102.22 & 0.00 & 23.66 \\
\hline 84.0 & 108.32 & 110.11 & 103.44 & 105.28 & 22.83 & 22.92 & -0.12 & 1.65 & 109.21 & 104.36 & 0.77 & 22.88 \\
\hline 89.0 & 103.57 & 106.32 & 101.42 & 103.03 & 15.35 & 11.66 & 0.52 & 2.04 & 104.94 & 102.22 & 1.28 & 13.51 \\
\hline 94.0 & 106.99 & 109.47 & 103.55 & 104.72 & 22.55 & 21.11 & -0.71 & 1.48 & 108.23 & 104.14 & 0.39 & 21.83 \\
\hline 99.0 & 107.51 & 105.32 & 105.15 & 103.20 & 14.88 & 18.70 & 1.36 & 1.35 & 106.41 & 104.18 & 1.36 & 16.79 \\
\hline
\end{tabular}


Table S4. Select normalized integral values of DCYA, compound 4a and products after addition of DBN (2,5-dimethylfuran was used as internal standard).

\begin{tabular}{|c|c|c|c|c|c|c|c|c|c|c|c|c|}
\hline \multirow{2}{*}{$\begin{array}{l}\text { Time } \\
(\min )\end{array}$} & \multicolumn{2}{|c|}{ 7,4'-oxyneolignan 4a } & \multicolumn{2}{|c|}{ DCYA } & \multicolumn{2}{|c|}{ Isoeugenol (1) } & \multicolumn{2}{|c|}{ DCYA adduct 2} & \multicolumn{4}{|c|}{ doEs (\%, average) } \\
\hline & $\begin{array}{c}\mathrm{H}-7 \\
\left(\delta_{\mathrm{H}}^{4.49-4.42)}\right.\end{array}$ & $\begin{array}{c}\mathrm{H}-5 \\
\left(\delta_{\mathrm{H}} 6.68-6.63\right)\end{array}$ & $\begin{array}{c}\mathrm{H}-1^{\prime} \\
\left(\delta_{\mathrm{H}} 3.10-3.05\right)\end{array}$ & $\begin{array}{c}\mathrm{H}-8 \\
\left(\delta_{\mathrm{H}} 8.31-8.28\right)\end{array}$ & $\begin{array}{l}\text { Half peak of } \mathrm{H}-7 \\
\left(\delta_{\mathrm{H}} 6.35-6.32\right)\end{array}$ & $\begin{array}{l}\text { Half peak of H-9 } \\
\left(\delta_{\mathrm{H}} 1.87-1.85\right)\end{array}$ & $\begin{array}{l}\text { Integral ratio of H-2 } \\
\left(\delta_{\mathrm{H}} 8.20-8.15\right)\end{array}$ & $\begin{array}{c}\text { Integral ratio of } \mathrm{H}-7 " \\
\left(\delta_{\mathrm{H}} 3.41-3.35\right)\end{array}$ & $\begin{array}{c}7,4^{-}- \\
\text {oxyneolignan } \\
\mathbf{4 a} \\
\end{array}$ & DCYA & $\begin{array}{c}\text { DCYA } \\
\text { adduct } 2\end{array}$ & Isoeugenol (1) \\
\hline 0.0 & 100.00 & 100.00 & 100.00 & 100.00 & 0.00 & 0.00 & -0.02 & 1.20 & 100.00 & 100.00 & 0.43 & 0.00 \\
\hline 5.0 & 80.66 & 82.96 & 74.20 & 77.91 & 18.80 & 5.69 & 28.02 & 30.46 & 81.81 & 76.05 & 29.75 & 11.90 \\
\hline 10.0 & 80.82 & 74.46 & 58.75 & 67.67 & 18.42 & 9.92 & 38.92 & 41.89 & 77.64 & 63.21 & 40.70 & 15.13 \\
\hline 15.0 & 73.57 & 67.59 & 56.08 & 58.65 & 26.67 & 17.88 & 48.29 & 50.79 & 70.58 & 57.37 & 50.48 & 23.18 \\
\hline 20.0 & 53.35 & 64.09 & 42.29 & 51.27 & 36.61 & 23.91 & 53.96 & 57.69 & 58.72 & 46.78 & 58.04 & 28.64 \\
\hline 25.0 & 43.68 & 63.22 & 45.46 & 48.48 & 43.30 & 42.41 & 63.55 & 68.66 & 53.45 & 46.97 & 67.87 & 39.90 \\
\hline 30.0 & 41.81 & 50.98 & 41.51 & 42.77 & 58.87 & 46.21 & 70.20 & 74.37 & 46.39 & 42.14 & 74.53 & 51.15 \\
\hline 35.0 & 59.11 & 50.93 & 34.81 & 35.83 & 50.85 & 39.72 & 72.08 & 78.38 & 55.02 & 35.32 & 77.04 & 46.52 \\
\hline 40.0 & 42.59 & 45.70 & 30.88 & 31.91 & 58.48 & 54.29 & 76.07 & 81.20 & 44.14 & 31.39 & 80.55 & 55.91 \\
\hline 45.0 & 41.09 & 49.93 & 21.31 & 28.68 & 54.53 & 51.90 & 81.29 & 87.22 & 45.51 & 25.00 & 86.24 & 51.88 \\
\hline 50.0 & 53.28 & 41.43 & 18.34 & 25.62 & 62.98 & 56.76 & 83.87 & 90.16 & 47.36 & 21.98 & 89.12 & 61.67 \\
\hline 55.0 & 36.65 & 33.50 & 22.47 & 23.15 & 70.91 & 63.56 & 87.42 & 93.06 & 35.08 & 22.81 & 91.57 & 67.71 \\
\hline 60.0 & 35.36 & 42.47 & 20.75 & 23.85 & 65.27 & 60.40 & 91.57 & 97.94 & 38.92 & 22.30 & 95.14 & 61.76 \\
\hline 65.0 & 47.50 & 36.70 & 18.83 & 19.46 & 64.10 & 58.66 & 89.36 & 95.94 & 42.10 & 19.14 & 93.14 & 63.01 \\
\hline 70.0 & 36.85 & 36.29 & 18.02 & 18.39 & 69.68 & 66.98 & 92.72 & 99.70 & 36.57 & 18.20 & 96.77 & 68.41 \\
\hline 75.0 & 23.02 & 31.54 & 16.42 & 17.78 & 81.79 & 74.55 & 95.60 & 101.17 & 27.28 & 17.10 & 98.83 & 76.88 \\
\hline 80.0 & 34.80 & 36.59 & 9.93 & 16.27 & 69.38 & 64.11 & 94.59 & 100.06 & 35.70 & 13.10 & 97.44 & 66.48 \\
\hline 85.0 & 24.78 & 39.46 & 9.55 & 16.01 & 72.28 & 64.08 & 94.74 & 101.33 & 32.12 & 12.78 & 97.76 & 65.96 \\
\hline 90.0 & 24.05 & 25.67 & 12.94 & 15.30 & 88.93 & 82.21 & 98.43 & 102.72 & 24.86 & 14.12 & 100.53 & 85.32 \\
\hline 95.0 & 31.94 & 27.70 & 8.02 & 14.48 & 78.98 & 76.59 & 97.74 & 102.57 & 29.82 & 11.25 & 100.00 & 78.43 \\
\hline 100.0 & 22.63 & 23.02 & 7.77 & 14.35 & 85.86 & 79.32 & 97.98 & 102.88 & 22.83 & 11.06 & 99.79 & 82.53 \\
\hline 105.0 & 38.10 & 34.05 & 11.06 & 14.09 & 74.28 & 68.48 & 100.12 & 105.23 & 36.08 & 12.57 & 100.66 & 72.00 \\
\hline 110.0 & 40.64 & 24.47 & 9.18 & 13.54 & 79.53 & 78.30 & 100.65 & 104.75 & 32.55 & 11.36 & 99.51 & 81.36 \\
\hline 115.0 & 28.77 & 21.74 & 10.04 & 12.55 & 86.37 & 76.99 & 98.69 & 102.09 & 25.25 & 11.29 & 99.96 & 82.74 \\
\hline 120.0 & 29.04 & 21.60 & 6.05 & 13.13 & 89.93 & 76.40 & 100.30 & 91.72 & 25.32 & 9.59 & 98.22 & 84.29 \\
\hline 125.0 & 27.81 & 25.01 & 5.77 & 12.41 & 85.29 & 78.90 & 101.45 & 106.68 & 26.41 & 9.09 & 102.90 & 82.52 \\
\hline 130.0 & 27.39 & 21.03 & 5.42 & 12.32 & 84.65 & 78.81 & 99.36 & 103.51 & 24.21 & 8.87 & 100.19 & 82.69 \\
\hline 135.0 & 26.98 & 24.48 & 5.45 & 11.50 & 83.34 & 82.54 & 100.58 & 106.08 & 25.73 & 8.47 & 101.94 & 83.32 \\
\hline 140.0 & 27.94 & 23.65 & 7.80 & 11.15 & 75.87 & 75.12 & 98.24 & 102.38 & 25.80 & 9.48 & 99.19 & 76.14 \\
\hline 145.0 & 14.77 & 19.12 & 3.88 & 11.31 & 89.12 & 85.43 & 99.32 & 102.66 & 16.94 & 7.59 & 100.20 & 86.61 \\
\hline 150.0 & 26.74 & 23.62 & 4.63 & 12.40 & 77.97 & 75.43 & 100.73 & 105.34 & 25.18 & 8.51 & 100.58 & 77.17 \\
\hline 155.0 & 13.43 & 19.08 & 3.36 & 10.77 & 90.94 & 86.90 & 99.94 & 103.56 & 16.25 & 7.06 & 100.60 & 88.07 \\
\hline 160.0 & 40.26 & 16.55 & 3.25 & 11.47 & 85.85 & 75.69 & 100.52 & 103.60 & 28.41 & 7.36 & 100.39 & 84.36 \\
\hline
\end{tabular}




\begin{tabular}{|c|c|c|c|c|c|c|c|c|c|c|c|c|}
\hline 165.0 & 14.77 & 22.05 & 6.91 & 10.86 & 81.47 & 82.40 & 100.30 & 105.13 & 18.41 & 8.88 & 101.52 & 80.83 \\
\hline 170.0 & 13.36 & 17.73 & 2.28 & 11.21 & 93.03 & 82.16 & 99.92 & 105.47 & 15.55 & 6.75 & 101.09 & 86.93 \\
\hline 175.0 & 11.30 & 17.35 & 3.01 & 11.15 & 93.25 & 88.31 & 100.49 & 102.33 & 14.33 & 7.08 & 100.27 & 89.87 \\
\hline 180.0 & 6.20 & 21.00 & 2.38 & 11.11 & 91.94 & 90.86 & 101.55 & 105.47 & 13.60 & 6.74 & 102.14 & 89.16 \\
\hline 185.0 & 15.39 & 17.19 & 1.90 & 10.98 & 95.10 & 90.74 & 102.84 & 107.73 & 16.29 & 6.44 & 103.44 & 92.65 \\
\hline 190.0 & 11.35 & 20.48 & 1.79 & 10.95 & 93.26 & 91.01 & 102.92 & 96.46 & 15.92 & 6.37 & 100.53 & 90.76 \\
\hline 195.0 & 11.37 & 13.45 & 2.17 & 10.71 & 96.69 & 93.38 & 103.31 & 108.74 & 12.41 & 6.44 & 104.09 & 94.72 \\
\hline 200.0 & 31.59 & 13.22 & 1.68 & 10.51 & 91.55 & 81.19 & 100.42 & 94.00 & 22.40 & 6.10 & 97.08 & 89.15 \\
\hline 205.0 & 9.60 & 14.03 & 2.29 & 10.35 & 95.68 & 83.09 & 100.17 & 96.22 & 11.82 & 6.32 & 97.68 & 88.71 \\
\hline 210.0 & 18.43 & 15.81 & 1.38 & 10.90 & 92.52 & 83.78 & 101.19 & 106.06 & 17.12 & 6.14 & 101.61 & 88.54 \\
\hline 215.0 & 8.26 & 12.75 & 1.86 & 11.16 & 99.08 & 91.67 & 101.92 & 97.88 & 10.51 & 6.51 & 99.92 & 94.69 \\
\hline 220.0 & 31.15 & 14.50 & 1.47 & 11.10 & 91.85 & 77.76 & 102.06 & 99.33 & 22.82 & 6.29 & 100.86 & 87.33 \\
\hline 225.0 & 21.47 & 15.42 & 1.30 & 10.76 & 92.55 & 83.26 & 101.56 & 98.55 & 18.44 & 6.03 & 99.54 & 88.82 \\
\hline 230.0 & 28.68 & 16.26 & 1.38 & 10.54 & 89.28 & 77.69 & 99.57 & 96.84 & 22.47 & 5.96 & 98.65 & 85.36 \\
\hline 235.0 & 12.96 & 16.92 & 1.51 & 10.92 & 91.79 & 83.36 & 99.87 & 106.52 & 14.94 & 6.21 & 100.99 & 86.98 \\
\hline 240.0 & 14.01 & 12.31 & 1.44 & 11.07 & 97.71 & 93.84 & 101.90 & 96.51 & 13.16 & 6.25 & 100.35 & 96.04 \\
\hline 245.0 & 14.59 & 11.82 & 1.41 & 11.04 & 96.33 & 94.84 & 100.00 & 100.00 & 13.20 & 6.22 & 100.00 & 96.01 \\
\hline
\end{tabular}

